\title{
Inferring directionality of coupled dynamical systems using Gaussian process priors: Application on neurovascular systems
}

\author{
Ameer Ghouse, ${ }^{1, *}$ Luca Faes, ${ }^{2, \dagger}$ and Gaetano Valenza ${ }^{1, *}$ \\ ${ }^{1}$ Bioengineering and Robotics Research Centre "E Piaggio" \& Department of Engineering, University of Pisa, 56122 Pisa, Italy \\ ${ }^{2}$ Department of Engineering, University of Palermo, 90133 Palermo, Italy
}

(Received 15 December 2020; accepted 9 November 2021; published 17 December 2021)

\begin{abstract}
Dynamical system theory has recently shown promise for uncovering causality and directionality in complex systems, particularly using the method of convergent cross mapping (CCM). In spite of its success in the literature, the presence of process noise raises concern about CCM's ability to uncover coupling direction. Furthermore, CCM's capacity to detect indirect causal links may be challenged in simulated unidrectionally coupled Rossler-Lorenz systems. To overcome these limitations, we propose a method that places a Gaussian process prior on a cross mapping function (named GP-CCM) to impose constraints on local state space neighborhood comparisons. Bayesian posterior likelihood and evidence ratio tests, as well as surrogate data analyses are performed to obtain a robust statistic for dynamical coupling directionality. We demonstrate GP-CCM's performance with respect to CCM in synthetic data simulation as well as in empirical electroencephelography (EEG) and functional near infrared spectroscopy (fNIRS) activity data. Our findings show that GP-CCM provides a statistic that consistently reports indirect causal structures in non-separable unidirectional system interactions; GP-CCM also provides coupling direction estimates in noisy physiological signals, showing that EEG likely causes, i.e., drives, fNIRS dynamics.
\end{abstract}

DOI: 10.1103/PhysRevE.104.064208

\section{INTRODUCTION}

Coupling statistics are crucial for describing the relationship between dynamical systems observed in physical data. Many traditional coupling estimation methods, such as correlation or mutual information measures, are by mathematical definition pairwise analysis of samples [1], thus encode no inherent causal temporal structure. While these measures have been prevalently used-for example, functional connectivity analysis of high dimensional spatial data [1-3] - they neglect to account for the dynamical structure of a time series, and related causal graphs can make no reference to directionality [2]. Among the coupling methods handling the dynamical structure of time series data, Granger causality is widely used [4]. Granger causality was initially introduced in the field of economics, e.g., to analyze economic growth [5,6], and was thereafter adopted by the neuroscience community for effective brain connectivity. In the framework of stochastic processes, the usage of the probabilistic notion underlying Granger causality increased even more with the introduction of its nonparametric counterpart, transfer entropy [7], which

\footnotetext{
*ameer.ghouse@studenti.unipi.it

$\dagger$ luca.faes@unipa.it

‡g.valenza@ing.unipi.it
}

Published by the American Physical Society under the terms of the Creative Commons Attribution 4.0 International license. Further distribution of this work must maintain attribution to the author(s) and the published article's title, journal citation, and DOI. has rapidly become a reference tool for effective connectivity analysis in network physiology [8,9]. In spite of the huge potential of tools like Granger causality and transfer entropy, the computation of the relevant measures is strongly influenced by issues including the effects of hidden or unobserved variables, the dependence on the temporal resolution through which the observed time series are sampled, the difficulty of operating in high-dimensional spaces, and the presence of observational noise $[10,11]$.

Another issue with Granger causality, raised, e.g., in Ref. [12], regards its assumption of nonseparability between the states being analyzed. Precisely, Granger causality is specified as a multivariate autoregressive model where states have no synergistic coupling (such as a quadratic coupling), an effect that is indeed commonly seen in complex systems [13]. An example of nonseparability in neurophysical systems particularly arise in the neurovascular system, as seen in the hemodynamic model, where deoxyhemoglobin has synergistic coupling with blood volume [14]. In addition, literature has shown that in the event of incompletely observed states, e.g., when there is error in the sampling time of events as common in planetary, geological or any other sciences where temporal resolution is low, GC (and transfer entropy) further fail $[15,16]$. Thus, ambiguous results can be obtained from Granger causality due to the difficulty to attain the condition of separability of the information about the putative cause of the time series from the dynamics of the effect series even if single fit models specified by state space parameters can be identified $[17,18]$. To overcome these limitations, convergent cross mapping (CCM) has been proposed as a method that treats two time series as deterministic signals, using em- 
bedding dynamical systems theory attempts to measure the extent that states from one series's reconstructed phase space maps onto another [12]. This formulation lends it powerful in detecting causality in such synergistically coupled systems in which Granger causality may fail. Despite its recent introduction, CCM has already shown several applications. For example, it has been applied in the analysis of functional brain connectivity during resting state for uncovering default networks [19], in several studies investigating how the cardiovascular system is coupled with the central nervous system through brain-heart interactions [20-24], and in describing ecological systems such as those relating anchovy population dynamics to sea-temperature and sardine population dynamics [12] or plant species richness and soil nitrate levels [25].

In spite of the successful use of CCM in previous research, some of its properties can limit its applicability in the analysis of general coupled dynamic systems. Particularly, this method assumes that the time series under investigation are generated from deterministic systems, thus CCM contains no consideration of process noise [26]. To overcome these limitations, we develop and demonstrate a new method for inferring causality between pairs of time series measured as outputs of potentially coupled dynamical systems. The method is formulated as a Bayesian model comparison between models of state spaces composed of states of one time series predicting the states of a second series, with zero-mean Gaussian process priors placed on each model. Furthermore, as CCM, the proposed method maintains the benefit that underpins CCM of being a nonparametric statistic, yielding its results primarily as a function of the whole data rather than parametric estimates.

Bayesian model evidence, particularly evidence lower bound methods, have been used in the past also for determining causal structures as in Ref. [27]. Typically, one specifies a set of forward models that may hypothetically explain the data. Note that we do not use such Bayesian models as we are primarily interested in causal inference in datasets where a physical model hypothesis does not immediately exist for the forward model. Other Bayesian models, called Bayesian networks, can be constructed using causal directed acyclic graphs that describe the causal flow between observables of of a system, such as the PC and PCMCI method [28,29]. These methods find the causality between two random variables, let us say $X$ and $Y$, by determining whether they are conditionally independent given a set of variables $S$. This process is repeated through all variables to create the originating causal directed acyclic graphs.

In regards to causal analysis methods based on interventions, as formalized by Judea Pearl [30], there have been some interesting frameworks such as the so called "dynamical causal effects" [31], which implements the interventional approach for stochastic dynamic systems. More precisely, this approach requires some experimental procedure to invoke causal interventions or a mathematical model to perform "virtual interventions" to see "how coupling manifests itself in the dynamics," a very curious question indeed given how it may be possible causal effects manifest differently between variables in different areas of state space space. This approach has even shown that transfer entropy and Granger causality are in fact particular instantiations of the dynamical causal effects framework [32]. Though this is an elegantly designed method for investigating causal interactions, we focus on causal discoveries in instances where we have no possible intervention framework to work in.

We must note that Gaussian processes have been investigated already for the sake of ameliorating the CCM algorithm as in Feng et al. [33]. Their method particularly tried to address an optimal reconstruction of the system's phase space by placing priors on the embedding dimension and lag time parameters, formulated using a maximum evidence lower bound in a variational Bayesian setting with a Gaussian process approximating variational distribution. Instead, we develop a new statistic that leverages the fact that we are provided with posterior probability distributions for the data with Gaussian processes which can be used for calculating evidence likelihood ratios for models [34]. Particularly, we aim to develop a statistic to reveal the presence and direction of a directional coupling between noisy dynamical systems, when unidirectionality is a priori assumed.

Here, we test the performance of the proposed Gaussian process convergent cross mapping (GP-CCM) in synthetic data gathered from coupled multidimensional systems, and in real multivariate data from brain recordings of electroencephelography (EEG) and functional near infrared spectroscopy (fNIRS) activity data. Simulations are used to assess the comparative ability of CCM and of the proposed method to identify coupling direction in bidirectionally and unidirectionally coupled dynamics, as well as the robustness to noise; such simulations are performed in benchmark systems such as the paradigmatic coupled logistic map investigated in and both simple and chaotic differential equation systems.

The application study in neurovascular systems involving fNIRS and EEG are particularly interesting to investigate as there exists a unique link between the metabolic signal as measured by fNIRS and the electrophysiological signals captured by EEG. In the case of EEG, signals arise as neurons activate, leading to ionic currents caused by polarization activity in the neuron through active and passive ion channels $[35,36]$; this movement of charged ions and their electric field give rise to an extracellular volume conduction effect whose volume integral is sensed by the electrode of the EEG array [37]. In fNIRS, with neuron activation the aforementioned active ionic channels demand oxygen for reduction reactions to produce ATP for metabolic purposes [38]. As this leads to a change in oxy- and de-oxyhemoglobin concentrations [39], fNIRS then operates on detecting changes in absorbtion of near infrared light mediated by changing concentrations of hemoglobin [40]. Considering the common thread of active ion channels being the genesis of both metabolic and electrophysiological signals, it begs the question of the coupling of the two systems through this link which we explore in this study.

\section{MATERIALS AND METHODS}

\section{A. Embedding techniques for reconstructing phase space}

Takens theorem $[41,42]$ permits a reconstruction of the state-space of a dynamical system $\mathcal{X}$ that generated an observed time series $X$ with samples $x_{i}$. The $i$ th state of the system $\mathcal{X}$ is represented by a $m_{x}$-dimensional delay coordinate 
embedding $\phi(X)_{i}$ as follows:

$$
\begin{aligned}
\phi(X)_{i} & =\left\{x_{i}, x_{i+\tau}, \ldots, x_{i+\left(m_{x}-1\right) \tau}\right\}, \\
i & \in 1,2, \ldots, N,
\end{aligned}
$$

where $N$ is the number of states observed, $\tau$ is the time-delay, and $m_{x}$ is the embedding dimension associated with time series $X$ for dynamical system $\mathcal{X}$. Given a time series with $N+\left(m_{x}-1\right) \tau$ samples, $\phi(X)$ results in a matrix in $\mathbb{R}^{N \times m_{x}}$, where each row is a state, and each column is a variable in the state space.

\section{B. Convergent cross mapping}

Introduced in Ref. [12] as a method to investigate coupling in weakly or moderately coupled complex systems with nonseparable dynamics, CCM was shown to perform well in complex system cases such as ecosystems problems to detect unidirectional coupling with robustness against false positives. CCM leverages Takens' theorem by considering whether the system that generates the time series $Y$ is causal to the system that generates the time series $X$ through the idea of cross-mapping, i.e., the delay coordinate map for $Y$ should cross map with high correlation to $X$ (see Appendix 1 for a visualization of this cross-mapping effect). The reverse may not be exactly true, however, for a delay coordinate map $\phi(Y)$ cross-mapping to $X$, as $\phi(Y)$ may not contain all the dynamical information of $\phi(X)$.

For testing whether system $\mathcal{Y}$ drives $\mathcal{X}$, a local region of the states $\phi(X)$ around a cross-mapped state $\phi(X)_{i}$ should be predictive of $y_{i}$. Formally, the prediction of $y_{i}$ given the states in $\phi(X)$ is:

$$
y_{i} \mid \phi(X)=\sum_{k=1}^{m_{x}} w_{i k} y_{t_{k}}
$$

where $t_{k}, k=1, \ldots, m_{x}$, are the $m_{x}$ time indices of the neighbors of $\phi(X)_{i}$ in $\phi(X)$ (sorted in ascending order by their distance to $\left.\phi(X)_{i}\right)$; according to [12], the weights $w_{i k}$ are determined by:

$$
w_{i k}=\frac{u_{i k}}{\sum_{j=1}^{m} u_{i j}},
$$

where

$$
u_{i k}=\exp \left\{-\frac{d\left[\phi(X)_{i}, \phi(X)_{t_{k}}\right]}{d\left[\phi(X)_{i}, \phi(X)_{t_{1}}\right]}\right\}
$$

with $d$ being the euclidean distance between two $m_{x}$ dimensional vectors. If $\mathcal{X}$ and $\mathcal{Y}$ are dynamically coupled, then the weighted sum in Eq. (2) should be predictive of $y_{i}$. This is determined by the Pearson's correlation coefficient:

$$
\rho_{Y \mid \phi(X)}=\frac{\operatorname{Cov}[Y, Y \mid \phi(X)]}{\sqrt{\operatorname{Var}(Y) \cdot \operatorname{Var}[Y \mid \phi(X)]}},
$$

where $\quad \operatorname{Cov}[Y, Y \mid \phi(X)]=\mathbb{E}\{(Y-\mathbb{E}[Y])(Y \mid \phi(X)-$ $\mathbb{E}[Y \mid \phi(X)])\}$ and $\operatorname{Var}(Y)=\mathbb{E}\left\{(Y-\mathbb{E}[Y])^{2}\right\}, \operatorname{Var}[Y \mid \phi(X)]=$ $\mathbb{E}\left\{(Y \mid \phi(X)-\mathbb{E}[Y \mid \phi(X)])^{2}\right\}$.

Reversing the role of the two time series in the analysis, one can compute the causal coupling from $X$ to $Y, \rho_{X \mid \phi(Y)}$. Then, the sign of the difference between $\rho_{X \mid \phi(Y)}$ and $\rho_{Y \mid \phi(X)}$ can be taken as indicative of the causal direction:

$$
\Delta=\rho_{X \mid \phi(Y)}^{2}-\rho_{Y \mid \phi(X)}^{2},
$$

with positive values being indicative of prevalent causal coupling from $X$ to $Y$.

\section{The proposed method: Gaussian process convergent cross mapping}

GP-CCM has its foundation in Gaussian process regression, which is a Bayesian regression technique where each point in a set of data is assumed to be sampled from a multivariate Gaussian distribution [43]. Before introducing the framework of Gaussian processes, we provide an illustrative example of obtaining posterior distributions given a bivariate joint probability distribution.

Let us consider two scalar random variables $X^{i}$ and $X^{j}$ that generate samples $x_{i}$ and $x_{j}$, respectively, where $x_{i}$ is considered as the observed state and $x_{j}$ is considered as the unobserved state. Then, the bivariate Gaussian joint probability density function between $X^{i}$ and $X^{j}$ is as follows:

$$
p\left(X^{i}, X^{j}\right)=\mathcal{N}(\mu, C)=\mathcal{N}\left(\left[\mu_{i}, \mu_{j}\right],\left[\begin{array}{ll}
C_{i i} & C_{i j} \\
C_{j i} & C_{j j}
\end{array}\right]\right),
$$

where $\mu$ and $C$ represent the mean vector and the covariance matrix of the two variables $X^{i}$ and $X^{j}$, with $C_{i i}=\mathbb{E}\left[\left(X^{i}-\right.\right.$ $\left.\left.\mu_{i}\right)^{2}\right]$ being the variance of $X^{i}$, and $\mu_{i}=\mathbb{E}\left[X^{i}\right]$.

The objective is then to find the conditional probability $p\left(X^{j} \mid X^{i}\right)$, which can be achieved by dividing the joint probability $p\left(X^{i}, X^{j}\right)$ by the marginal Gaussian probability density function:

$$
p\left(X^{i}\right)=\int_{-\infty}^{\infty} p\left(X^{i}, X^{j}\right) d X^{j}=\mathcal{N}\left(\mu_{i}, C_{i i}\right) .
$$

A known result in linear Gaussian regression is that $X^{j} \mid X^{i}$ is a Gaussian random variable with mean $\mu_{X^{j} \mid X^{i}}$ and variance $\sigma_{X^{j} \mid X^{i}}^{2}$ depending on $\mu$ and $C$ :

$$
\begin{aligned}
p\left(X^{j} \mid X^{i}\right) & =\mathcal{N}\left(\mu_{X^{j} \mid X^{i}}=\mu_{j}+C_{j i} C_{i i}^{-1}\left(X^{i}-\mu_{i}\right),\right. \\
\sigma_{X^{j} \mid X^{i}}^{2} & =C_{j j}-C_{j i} C_{i i}^{-1} C_{i j} .
\end{aligned}
$$

\section{Formulation of the proposed method}

In our work, Gaussian processes generalize the aforementioned statistical framework into functional spaces where the mean function is considered null a priori and the a priori covariance function $K[\phi(X), \phi(X)]$ is referred to as the "kernel" function. $\phi(X)$ and $\phi(Y)$ are considered realizations of the random Gaussian process. The kernel is a priori selected as a squared exponential covariance with automatic relevance determination [44] with hyperparameters $\theta_{x}^{\text {ard }}=\left\{A, l_{1}, l_{2} \ldots l_{m_{x}}\right\}$ :

$$
K\left[\phi(X)_{i}, \phi(X)_{j}\right]=A e^{-\sum_{n=1}^{m_{x}} \frac{\left|\phi(X)_{i_{n}}-\phi(X)_{j_{n}}\right|_{2}^{2}}{2 l_{n}^{2}},}
$$

where $\phi(X)_{i_{n}}=x_{i+(n-1) \tau}$ and $\phi(X)_{j_{n}}=x_{j+(n-1) \tau}$ are the $n$th scalar components of $\phi(X)_{i}$ and $\phi(X)_{j}$. The kernel function defined in Eq. (10) returns a scalar value quantifying the distance between the two states $\phi(X)_{i}$ and $\phi(X)_{j}$ in analogy to how CCM computes the weights in Eqs. (3) and (4). When evaluated for all possible pairs of observed states $\phi(X)_{i}$ and 
$\phi(X)_{j}, i, j=1, \ldots, N$, the kernel function forms the $N \times N$ matrix $K_{x}=\left\{K\left[\phi(X)_{i}, \phi(X)_{j}\right]\right\}$, which represents an autocovariance on the state space $\phi(X)$. Similarly, we can form a kernel matrix for the states in $Y, K_{y}=\left\{K\left[\phi(Y)_{i}, \phi(Y)_{j}\right]\right\}$, and a "cross" kernel matrix $K_{x y}=\left\{K\left[\phi(X)_{i}, \phi(Y)_{j}\right]\right\}$, representing, respectively, the autocovariance on the state space $\phi(Y)$ the cross-covariance between the state spaces $\phi(X)$ and $\phi(Y)$; assuming that $\phi(X)$ and $\phi(Y)$ have the same number of states $N$, all kernel matrices have dimension $N \times N$.

Thus, the Gaussian process framework allows us to place a Gaussian probability density function at each point on the reconstructed state space. Consequently, the concept of crossmapping turns into a posterior probability analysis of the likelihood $p(\phi(Y) \mid \phi(X))$. To develop a causal metric where $\mathcal{Y}$ drives $\mathcal{X}$, we first create a multivariate Gaussian distribution from the Gaussian Process model:

$$
p\left[\phi(X), \phi(Y) ; \theta_{x}^{a r d}, j\right]=\mathcal{N}\left([\mathbf{0}, \mathbf{0}],\left[\begin{array}{cc}
K_{x} & K_{x y} \\
K_{y x} & K_{y}
\end{array}\right]\right),
$$

where the means $\mathbf{0}$ are zero vectors with length equivalent to the number of states in $\phi(X)$ or $\phi(Y), \mathrm{j}$ specifies the variable of state space, and $K_{x}, K_{y}$, and $K_{x y}$ are the auto- and crosscovariance matrices defined above $\left(K_{y x}=K_{x y}^{\mathrm{T}}\right)$. Considering that the squared exponential kernel is defined as a distance metric over states, it can be considered stationary. This can be proven by showing how $K\left[\phi(X)_{i}, \phi(X)_{i}\right]$ will always be a scalar $A$ given this kernel, thus the prior Gaussian marginal statistics are the same over all data for each data point. Furthermore, due to how it operates over multidimensional state space, the kernel function can be seen as quantifying the spatial covariance in state space.

Kernels can be further tailored if we have more prior information on the data, e.g., if we have prior knowledge that i.i.d. white noise with zero mean and standard deviation $\sigma_{x}$ is added on each observation (i.e., in our problem, on the state variables).

If we are only concerned about points $\phi(X)$ and $\phi(Y)$ and we are developing a posterior model for the likelihood of $\mathcal{Y}$ GP-CCM causing $\mathcal{X}$, then $\sigma_{x}$ can be prior knowledge of noise on $\phi(X)$, i.e., a zero-mean Gaussian with a diagonal covariance matrix on elements corresponding to $\phi(X)$, i.e. $\Sigma=\left[\begin{array}{cc}\sigma_{x} I & 0 \\ 0 & 0\end{array}\right]$. As a consequence, we augment Eq. (11) as follows:

$$
\begin{aligned}
p & {\left[\phi(X), \phi(Y) ; \theta_{x}^{\text {ard }}, \sigma_{x}, j\right] } \\
& =p\left[\phi(X), \phi(Y) ; \theta_{x}^{\text {ard }}, j\right] p\left[\phi(X), \phi(Y) ; \sigma_{x}, j\right] \\
& =\mathcal{N}\left([\mathbf{0}, \mathbf{0}],\left[\begin{array}{cc}
K_{x}+\sigma_{x} I & K_{x y} \\
K_{y x} & K_{y}
\end{array}\right]\right) .
\end{aligned}
$$

Let us have $\theta_{x}$ be a set of all kernel parameters and $K^{\theta_{x}}$ denote our composite kernel. To find optimal parameters we maximize the log marginal likelihood that $\phi(X)$ is a zeromean Gaussian process over all variables $j$. Note that if instead one has knowledge of what the hyperparameters can be, then the optimization step can be skipped. The optimal point estimate of kernel parameters is denoted as $\theta_{x}^{*}$. From here, we can obtain the posterior distribution for $\phi(Y)$ using the rule we learned earlier in Eq. (9):

$$
\begin{aligned}
p\left[\phi(Y) \mid \phi(X) ; \theta_{x}^{*}, j\right] & =\mathcal{N}\left[\boldsymbol{\mu}_{\boldsymbol{y} \mid x, j}=K_{y x}^{\theta_{x}^{*}} K_{x}^{\theta_{x}^{*}-1} \phi(X)_{\cdot j}\right], \\
\Sigma_{y \mid x, j} & =K_{y}^{\theta_{x}^{*}}-K_{y x}^{\theta_{x}^{*}} K_{x}^{\theta_{x}^{*}-1} K_{x y}^{\theta_{x}^{*}} .
\end{aligned}
$$

Similarly we can obtain $p\left[\phi(X) \mid \phi(Y), \theta_{y}^{*}, j\right]$. These probabilities draw the causal strength statistics: $p[\phi(Y) \rightarrow$ $\phi(X)]=\prod_{j=1}^{m_{x}} p\left[\phi(Y) \mid \phi(X), \theta_{x}^{*}, j\right] ; \quad p[\phi(X) \rightarrow \phi(Y)]=$ $\prod_{j=1}^{m_{y}} p\left[\phi(X) \mid \phi(Y), \theta_{y}^{*}, j\right]$. We determine the most likely causal direction by a Bayesian model evidence comparison (BF) test:

$$
\mathrm{BF}=\frac{\sup \left\{\prod_{j=1}^{m_{x}} p\left[\phi(X) \mid \phi(Y) ; \theta_{x}^{*}, j\right]\right\}}{\sup \left\{\prod_{j=1}^{m_{y}} p\left[\phi(Y) \mid \phi(X) ; \theta_{y}^{*}, j\right]\right\}} .
$$

Considering $\phi(X)$ and $\phi(Y)$ are realizations of multivariate Gaussian distributions, $p\left[\phi(X) \mid \phi(Y) ; \theta_{x}^{*}, j\right]$ and $p\left[\phi(Y) \mid \phi(X) ; \theta_{y}^{*}, j\right]$ result in scalar values. From the logarithm of $\mathrm{BF}$ a statistic for the strength of evidence for causality can be found in one direction or the other. When BF is positive, there is greater evidence for $\mathcal{Y}$ driving $\mathcal{X}$. Negative $\mathrm{BF}$ indicates the opposite direction. Furthermore, a hyperbolic tangent can be taken on the log evidence comparison:

$$
\kappa=\tanh [0.5 \log (\mathrm{BF})] .
$$

This operation restricts the statistic to an open interval of $(-1,1)$ such that $|\kappa| \geqslant 0.5$ corresponds to a $\mathrm{BF} \geqslant 3$, i.e., substantial evidence as noted in prior literature in Ref. [34].

To assess the statistical significance of the proposed GPCCM estimate $\kappa$, i.e., to reject the null hypothesis that the GP-CCM result occurred from an acausal distribution, a surrogate analysis was performed. Particularly, acausal pairs of time series $X$ and $Y$ were generated by shuffling (i.e., permuting) the time points series of the original time series, thus destroying eventual temporal coupling structure of the time series. Performing GP-CCM on this permutation dataset provides a distribution of the GP-CCM statistic for temporally uncoupled time series that share the histogram of the original coupled time-series through which we can perform statistical significance tests. An $\alpha$ of 0.05 is used to determined whether the true GP-CCM directional coupling value is outside the 95\% confidence interval of the surrogate distribution, the statistic $\kappa$ is deemed significant for discriminating the predominant direction of coupling, i.e., predominant direction is $Y \rightarrow X$ if positive, otherwise directionality is $X \rightarrow Y$.

To summarize, the step-by-step instructions to obtain the proposed statistic are as follows:

(1) Reconstruct state spaces $\phi(X)$ and $\phi(Y)$.

(2) Find kernel parameters that maximize the marginal log likelihoods for each time series.

(3) Obtain posterior probability distributions for time series $X$ and $Y$ to obtain causal strength between series.

(4) Perform Bayesian model comparison to obtain statistic on substantiality of whether one time series GP-CCM causes another, i.e., causally influences the other.

(5) Perform a surrogate data analysis on $\kappa$ to assess the reliability of the estimate.

We note that exact modeling with Gaussian process priors may be a strong assumption. Furthermore, input to kernels are noisy as well. The next section introduces a nonstationary kernel that may potentially augment the model in such cases that 
TABLE I. Parameters of the systems.

\begin{tabular}{lc}
\hline \hline Logistic Maps & \\
\hline$R_{x}$ & 3.8 \\
$R_{y}$ & 3.55 \\
Lorenz & \\
$\sigma_{L}$ & $10^{-6}$ \\
$d t$ & $0.001 \mathrm{~s}$ \\
$\sigma$ & 10 \\
$\beta$ & $\frac{8}{3}$ \\
$\rho$ & 28 \\
Rossler & \\
$\sigma_{R}$ & 0.005 \\
$d t$ & $0.001 \mathrm{~s}$ \\
$\omega_{1}$ & 1.015 \\
$\omega_{2}$ & 0.985 \\
$\mathrm{a}$ & 0.15 \\
$\mathrm{~b}$ & 0.2 \\
$\mathrm{c}$ & 10 \\
$\mathrm{RL}$ Circuit & \\
$d t$ & $0.01 \mathrm{~s}$ \\
$\mathrm{R}$ & $1 \mathrm{k} \Omega$ \\
Realizations & 100 \\
\hline \hline
\end{tabular}

may help finding critical points in state space to determining a more robust posterior distribution.

\section{Sparse kernel representation}

It is known that taking the inverse of the kernel is an operation of computational cost $O\left(N^{3}\right)$, which can be prohibitive for large datasets. Furthermore, storage cost scales at $O\left(N^{2}\right)$. Beyond this, inputs to the kernel can be contaminated by noise. To act against these limitations, we propose a sparse kernel approximation as in Ref. [45] to obtain a set of "pseudoinputs" $P$ in sample space $K \ll N$, where $\mathrm{K}$ is the dimensionality of the pseudoinputs. The key to this is leveraging the following Nystrom approximation:

$$
K_{n} \approx \tilde{K}_{n}=K_{n k} K_{k}^{-1} K_{k n}
$$

The set of pseudoinputs $P$ in $\mathbb{R}^{m_{x} \times k}$ are then assumed as hyperparameters in $\theta$ as discussed previously, to be learned by maximum marginal likelihood. To assure that the learned pseudopoints are well representative of the data, a parameter $\Lambda=\operatorname{diag}(\lambda) ; \quad \lambda_{n n}=K_{n n}-k_{n}^{T} K_{k}^{-1} k_{n}$ is used to determine how well the sparse kernel represents true marginal statistics. Consequently, the marginal likelihood function is expressed as

$$
p\left[\phi(X) ; \theta_{x}, j\right]=\mathcal{N}\left(0, K_{n k} K_{k}^{-1} K_{k n}+\Lambda+\sigma_{x} I\right) .
$$

From Eq. (17), the Bayes rule is used as previously to obtain the posterior distribution $p[\phi(Y) \mid \phi(X), j]$. This solution reduces the computational cost drastically to $O\left(K N^{2}\right)$, and the storage cost to $O(K N)$. Furthermore, sparse kernels exploit a rich history for parameterizing latent noise-in-variable regression models [46-48] to learn a set of pseudopoints that best represent the data. These set of pseudopoints turns the marginal distribution nonstationary as seen by the $\Lambda$ param- eter being dependent on distance to pseudopoints which has much lower sample dimensionality than the original dataset.

\section{Experiments}

The experiments performed to validate GP-CCM are divided between simulations using coupled systems in deterministic chaos, and real-world data from physiological systems. All parameters for the simulations are defined in Table I. For the sake of consistency, all simulated time series were of length 3000 samples regardless of their integration time steps. When reconstructing state space, the embedding time delay is taken to be the first minimum of the averaged autocorrelation function [49]. As for the embedding dimension, it is taken to be the supremum of the optimal embedding dimensions for the two time series obtained by the false nearest neighbor algorithm [49]. The supremum is used based on Takens' embedding theorem discussed in Sec. II A stating that a minimum of the optimal embedding dimension for reconstructing a system's state space is twice the box counting dimension.

\section{Simulations}

a. Bidirectionally coupled logistic map. GP-CCM is first benchmarked against CCM using bidirectional logistic maps without the presence of process noise, as illustrated in the original work introducing CCM [12], to compare the ability of the two approaches in evaluating causal influence and determining coupling direction. The coupled bidirectional logistic maps take on the following form:

$$
\begin{aligned}
& X(t+1)=X(t)\left[R_{x}-R_{x} X(t)-B_{y x} Y(t)\right], \\
& Y(t+1)=Y(t)\left[R_{y}-R_{y} Y(t)-B_{x y} X(t)\right],
\end{aligned}
$$

where the parameters $B_{x y}$ and $B_{y x}$ control the coupling strength along the directions $X \rightarrow Y$ and $Y \rightarrow X$, respectively, and are here varied from 0 to 1 to simulate different conditions of coupling strength and coupling direction. $R_{x}$ and $R_{y}$ are parameters that control which regime of the bifurcation plot the logistic maps are. Their values, seen in Table I, were chosen such that they are in the chaotic regime.

b. Unidirectionally coupled attractors with process noise. The second simulation considers the coupling between a Lorenz system and a Rossler system. The formulation of the two systems driven by a Wiener process is reported in Eq. (19) for the Lorenz System and Eq. (20) for the Rossler system:

$$
\begin{aligned}
& d X_{1}=\left[\sigma\left(X_{2}-X_{1}\right)\right] d t+\sigma_{L}(t) d W_{X 1}, \\
& d X_{2}=\left[X_{1}\left(\rho-X_{3}\right)-X_{2}\right] d t+\sigma_{L}(t) d W_{X 2}, \\
& d X_{3}=\left(X_{1} X_{2}-\beta X_{3}\right) d t+\sigma_{L}(t) d W_{X 3}, \\
& d Y_{1}=\left(-\omega_{2} Y_{2}-Y_{3}\right) d t+\sigma_{R}(t) d W_{Y 1}, \\
& d Y_{2}=\left(\omega_{2} Y_{1}+a Y_{2}\right) d t+\sigma_{R}(t) d W_{Y 2}, \\
& d Y_{3}=\left[b+Y_{3}(Y 1-c)\right] d t+\sigma_{R}(t) d W_{Y 3} .
\end{aligned}
$$

The variable $\mathrm{W}$ stands for the Wiener process, whose generalized time derivative is a zero-mean i.i.d. process, i.e., $\mathcal{N}(0, I)$. Each state is integrated with their own independent Wiener process. The diffusion terms of the stochastic 


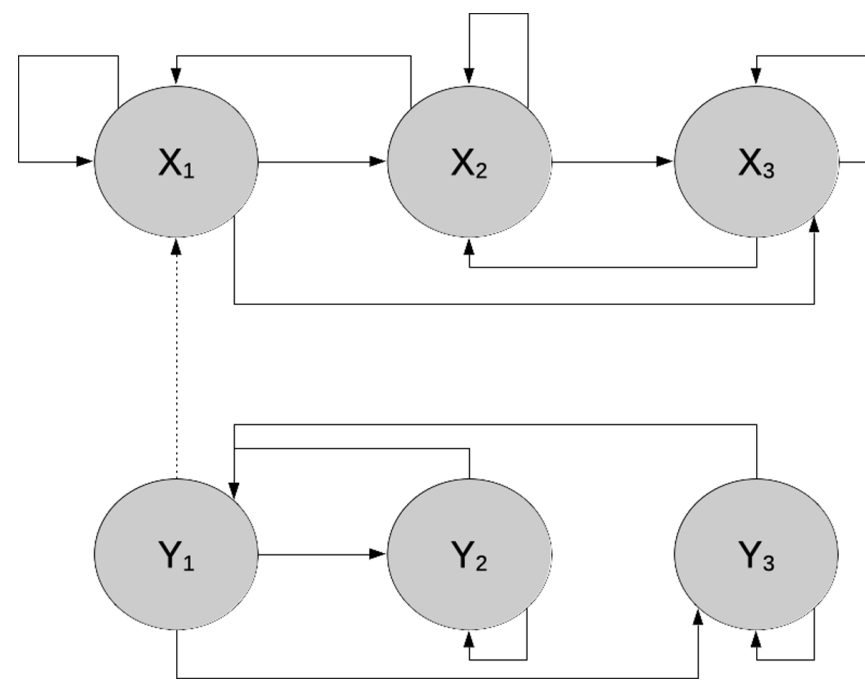

FIG. 1. Causal graph depicting the interaction between variables of the coupled Lorenz and Rossler systems. The direction and strength of coupling between the two systems is modulated by the parameter $\epsilon$ in Eqs. (21) and (22). Notice the indirect causal connection from $X_{2}$ and $X_{3}$ in the Lorenz system to $Y_{1}$ via the connection from $X_{1}$, and vice versa the indirect causal connection from $Y_{2}$ and $Y_{3}$ to $X_{1}$ mediated by $Y_{1}$.

differential equations were made a constant term for one set of experiments, and time varying linearly [e.g., $\sigma_{R}(t)=\sigma_{R} t$ ] for another set of experiments to check the effects of stationary and nonstationary diffusion on the causality metrics. The values of the parameters for the Rossler and Lorenz systems are shown in Table I. These parameters are selected in order that the systems are in deterministic chaos when undriven by external forcing terms.

To couple the systems, an additive term with form $\eta=$ $\epsilon A_{1}\left(1-B_{1}\right)$ can be used, where $A_{1}$ is the first state of the driving system, and $B_{1}$ is the first state of the driven system. Such a coupling function was used to ensure nonseparability of forcing terms. For a Lorenz system being driven by a Rossler system, Eq. (19) for $d X$ turns into the following:

$$
d X_{1}=\left[\sigma\left(X_{2}-X_{1}\right)+\epsilon X_{1}\left(Y_{1}-1\right)\right] d t+\sigma_{L}(t) d W_{X 1} .
$$

Rossler driven by Lorenz has the following form:

$$
d Y_{1}=\left[\omega_{2} Y_{2}-Y_{3}+\epsilon Y_{1}\left(X_{1}-1\right)\right] d t+\sigma_{R}(t) d W_{Y 1} .
$$

A causal graph of the interactions set for the two systems can be seen in Fig. 1.

c. RL circuit with process noise. A simple equation for an RL circuit is used to see if results of CCM and GP-CCM are consistent to determine coupling direction for coupled linear deterministic processes (voltage and current) in the presence of stochastic noise. The system has equations:

$$
\begin{aligned}
& V_{t}=\sin (\omega t), \\
& d I=\left(\frac{V_{t}}{L}-\frac{R}{L} I_{t}\right) d t+\sigma_{I} d W_{I},
\end{aligned}
$$

where $W$ is a similar Wiener process as before describing noise on the electrical current dynamics with sigma ${ }_{I}$ used as a scalar for the variance of the stochastic dynamics, which we tune in our experiments. The resistance parameter $R$ is defined in Table I.

\section{Physiological data}

The proposed real-data application regards the investigation between the electrical and hemodynamic activities of the brain, monitored, respectively, by EEG and fNIRS recordings.

a. Theory of EEG and hemodynamic coupling. For a given voxel (i.e., the elementary brain unit), the dynamics of transmembrane potential (hereafter called "neural activity") can be expressed as

$$
d v=-J v,
$$

where $v$ is a vector of neural activity in a local region, and $J$ is a square Jacobian matrix describing the effective connectivity between neurons. This is derived from a first-order approximation of an integrate and fire process described as

$$
d v=-\frac{v}{\tau}+u=f(v)
$$

where $\tau$ is the relaxation time constant coefficient, and $\mathrm{u}$ is a nonlinear function describing depolarization of neural activity ([u=g(v)]. Then, the EEG signal is derived from a lead-field row vector describing the linear mixture of neural activity to an electrode:

$$
e=l v \text {. }
$$

If we take the Samelson inverse of the lead-field vector, equivalent to the Moore-Penrose pseudoinverse of a vector where $l^{-1}=\frac{\bar{l}}{\|l\|^{2}}$, with the bar denoting complex conjugate and $\|\cdot\|$ denoting vector magnitude, then the electrical source activity can be defined from the EEG signal as follows:

$$
v=\frac{e \bar{l}}{\|l\|^{2}} .
$$

However, the hemodynamic signal for a voxel is proportional to the time expectation of electrical activity, according to

$$
B \propto c\left\langle v^{T} J v\right\rangle+\epsilon_{b}
$$

where $c$ is the transmembrane capacitance and a (possibly heteroskedastic) noise term $\epsilon_{b}$ is added to the model of hemoglobin activity as we can expect hemoglobin being driven by other factors such as glial cell metabolic demand. Substituting (27) into (28), we can see are directly caused by EEG in this model:

$$
B \propto c\left\langle\frac{e^{2}}{\|l\|^{4}} \bar{l}^{T} J \bar{l}\right\rangle+\epsilon_{b} .
$$

b. Data description and processing. Data taken from an open dataset [50] was used to assess the directional coupling between EEG band powers and fNIRS signals. Twenty-nine subjects were recruited for this study (aged 28.4 \pm 3.7 years old, 15 female). The experiment explored trials of either mental arithmetic activity compared to a baseline jointly or motor imagery tasks. There were 10 repetitions of either mental arithmetic, baseline activity, left-hand and right-hand motor imagery tasks per trial and six trials in sum. To study coupled hemodynamic and electrical activity, a careful selection of the 


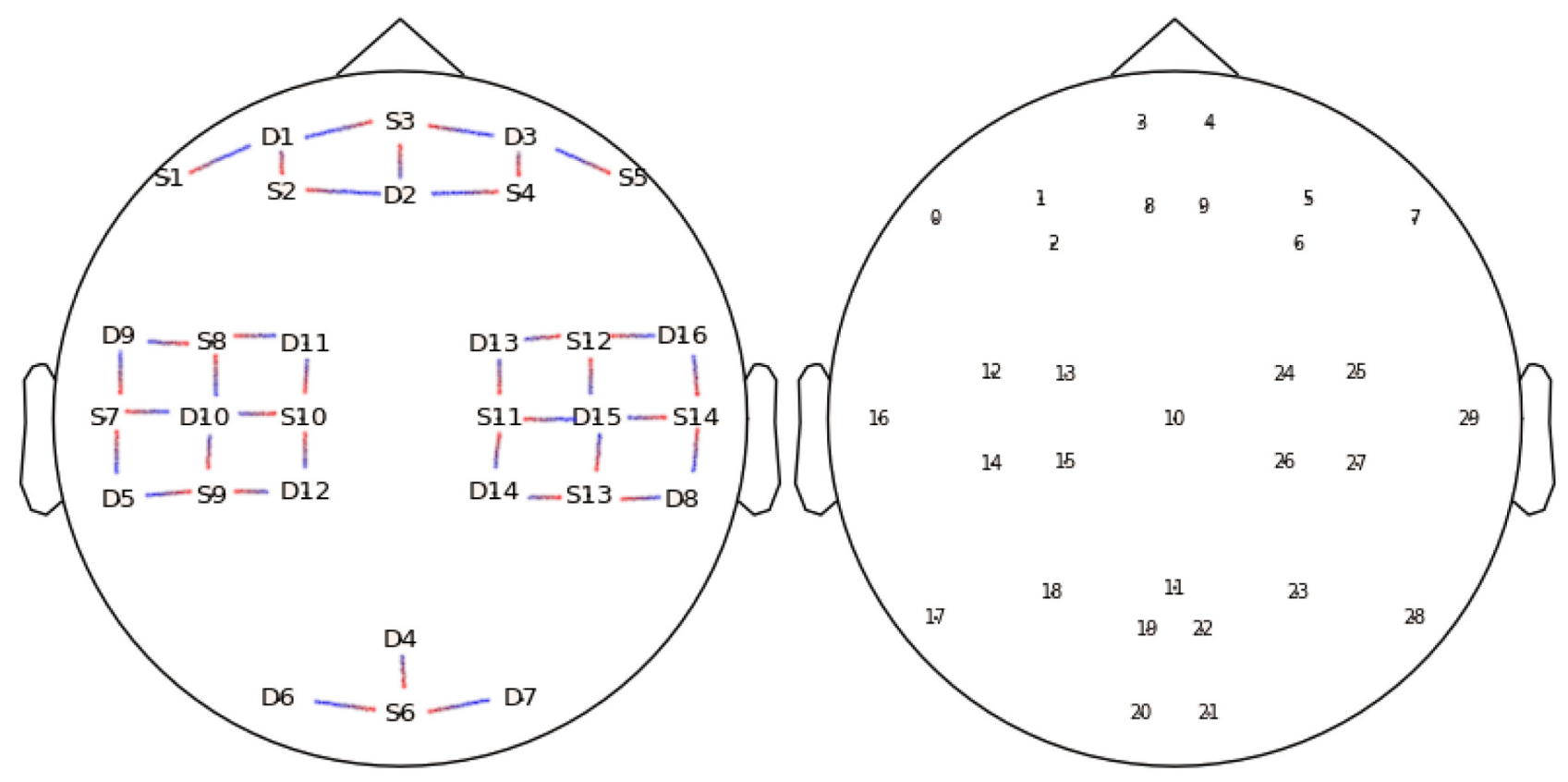

(a)

(b)

FIG. 2. Position of the optodes to resolve fNIRS information are seen in panel (a). Positions of the EEG electrodes are in panel (b). The four nearest EEG neighbors of D10 $(12,13,14,15)$, D15 $(24,25,26,27)$, D2 $(3,4,8,9)$, and D4 $(11,10,12,18)$ were taken to analyze coupling with respect to spatial regions.

signals measured from the EEG electrodes and fNIRS optodes positioned as shown in Fig. 2 must be performed.

There were 36 fNIRS channels in total derived from each source detector coupling (lines in Fig. 2). The fNIRS signals selected for the analysis were those recorded at optodes D10, D15, D2, and D4, representative of left-central, rightcentral, frontal, and posterior scalp regions. These signals, sampled at $10 \mathrm{~Hz}$, were frequency filtered using a sixth-order Butterworth filter to extract the bands lower than $0.6 \mathrm{~Hz}$ to capture hemodynamic response activity [51] and between 0.8 and $2 \mathrm{~Hz}$ to focus on pulsatile cardiac dynamics [52]. Each filter was applied in the forward and backward directions to obtain an overall zero-phase filter. Moreover, a wavelet filtering procedure was performed to attenuate artifacts due to motion, applying wavelet decomposition with a Daubechies five mother wavelet with nine decomposition levels; the detail coefficients with low probabilities were thresholded before reconstructing the hemoglobin signals.

Thirty EEG electrodes were acquired per subject and trial in the dataset. The EEG signals, sampled at $200 \mathrm{~Hz}$, were band pass filtered using a Butterworth filter of order 6 with a passband 0.5 to $50 \mathrm{~Hz}$; filtering was performed in both forward and reverse direction to obtain zero-phase. Channels with signal energy outside a $99 \%$ confidence interval were marked as bad channels [53]. Considering EEG is likely to be contaminated from extraneous noise sources - such as muscle activity, electrocular signals, electrocardiogram signals-algorithms for artefact reduction need to be used. Each signal was then decomposed using independent component analysis (ICA). The number of ICA components was determined by using the number of principal components that explained $99 \%$ of the variance. From there, $20 \mathrm{~s}$ moving windows with no overlap were taken for each ICA component. Similar to Ref. [54], features such as kurtosis, spectral entropy, skewness, logAlpha power, and $\frac{1}{f}$ fit were extracted from each window. These features were then taken to construct a multidimensional histogram, to reject windows in the ICA components that were of low probability. The artefact cleaned ICA components were then transformed back to the original EEG electrode space, and bad channels were spatially interpolated from the good channels using an inverse distance weighting method [53].

The nearest four EEG electrodes were matched with D10, D15, D2, and D4 optodes as seen in Fig. 2. From there, windowed 1D Fourier transforms were performed on each of the four electrodes with windows of $5 \mathrm{~s}$ and stride of $0.1 \mathrm{~s}$, resulting in a series of windowed fourier transforms of the EEG signals at intervals of $0.1 \mathrm{~s}$, or in other words a short time fourier transform (STFT). For each FFT, the power of the $\alpha(8-14 \mathrm{~Hz}), \beta(15-32 \mathrm{~Hz}), \gamma(32-50 \mathrm{~Hz}), \theta(4-8 \mathrm{~Hz})$, and $\delta(1-4 \mathrm{~Hz})$ bands [55] was calculated, thus resulting in a time course for each EEG band power sampled at $10 \mathrm{~Hz}$, i.e., the frequency of the fNIRS time series. Finally, for each band, the average of the EEG bandpower across the four nearest neighbors of an optode was taken to have a single time series to match with a single fNIRS signal.

c. Statistical analysis. First, the analysis was performed using the entire 10 minute time series for each trial performed for each subject to assess the causal coupling between the EEG bandpowers and the fNIRS signal. A second analysis was then executed after separating the 10 min series into time windows corresponding the tasks, to assess the causal coupling specifically during each task window. After that, the four brain regions were taken into consideration and an analysis was performed to see how coupling changes with respect to region for each task. 


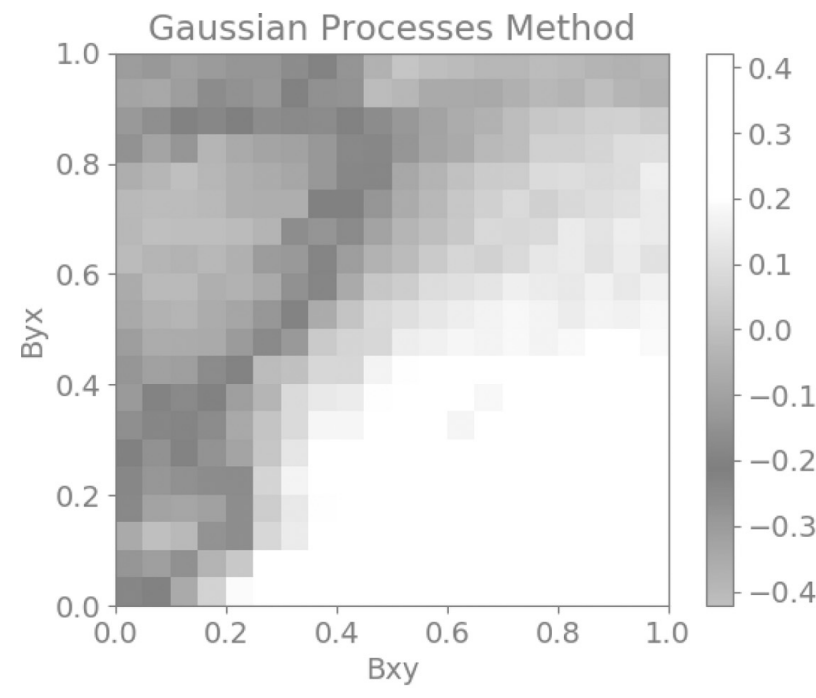

(a)

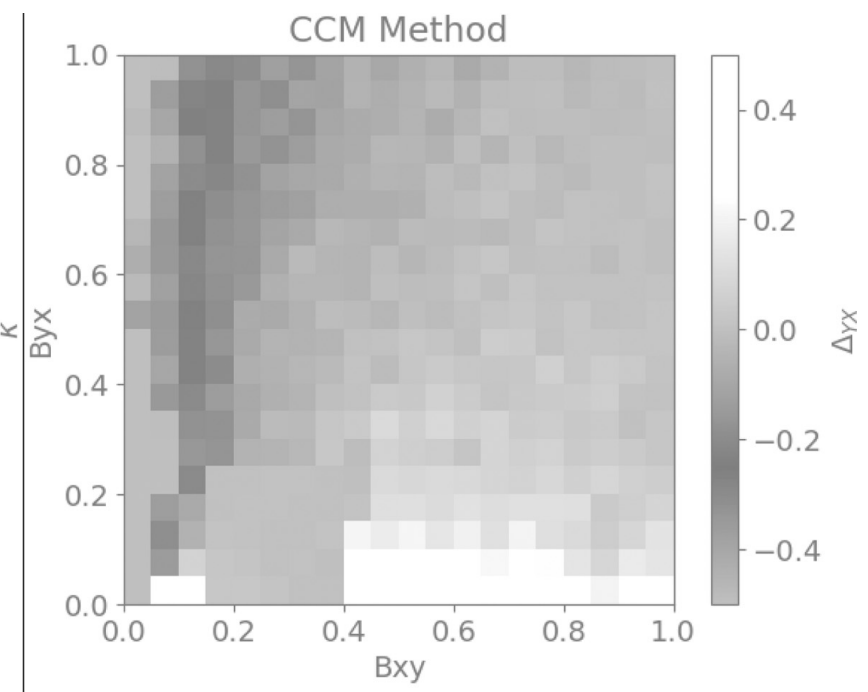

(b)

FIG. 3. Heatmaps of coupling statistic for varying coupling strength in the coupled logistic maps for CCM (b) and the poprosed method GP-CCM (a). White signifies stronger evidence for $X$ driving $Y$ while black signifies the reverse.

The Wilcoxon paired nonparametric statistical test was used to compare the distribution of results for leading causal driver (i.e., $\kappa$ in the proposed method, or $\Delta$ for $\mathrm{CCM}$ ) to see which EEG bandpowers were statistically different from each other for their statistic of coupling direction with fNIRS. Further Wilcoxon tests were performed to compare distributions of negative log likelihoods or CCM $\rho_{X \mid Y}$ score to see which EEG bands are different when describing causal strength with fNIRS.

To assess the statistical significance of the actual GPCCM method, surrogate analysis was performed. Particularly, acausal pair of time series were generated by shuffling (i.e., permuting) the time points of the original coupled time series, thus destroying temporal structure of the time-series for testing the null hypothesis that there is no causal relationship, i.e., performing GP-CCM on this permutation dataset provides a distribution of the GP-CCM statistic for an acausal time series that share the histogram of the original coupled time-series from which we can thus perform statistical significance tests with. An $\alpha$ of 0.05 was used to determined whether the true GP-CCM value is outside the $95 \%$ confidence interval of the bootstrapped distribution, thus $p<0.05$ states the statistic is significant. For the physiological time series, only statistically significant data points are shown in the boxplots below (Figs. 7 and 8). For the unidirectional coupled Rossler and Lorenz systems, we show an example of how a significance test can look like in the appendix Fig. 13.

\section{RESULTS}

\section{A. Simulations}

\section{Bidirectionally coupled logistic map}

It can be seen in Fig. 3 that when systems are strongly bidirectionally coupled (both $B_{x y}$ and $B_{y x}$ are high), convergent cross mapping has difficulty determining the coupling direction. On the contrary the method we propose is further capable to elicit the coupling direction in this regime (the white and black areas extend closer to the diagonal in Fig. 3), while still providing similar results as CCM in the cases when one parameter is clearly stronger. However, a factor that needs to be addressed is the fact that the substantiality is not reached as defined in Eq. (15).

a. Unidirectionally coupled attractors with process noise. Results from the Rossler system driving Lorenz are shown in Fig. 4 as a function of the coupling parameter $\epsilon$. For $\epsilon=0$, there is no substantial evidence of one system driving another in either CCM and GP-CCM, although GP-CCM provides estimates with deviation from zero larger than CCM. However, as the magnitude of $\epsilon$ increases, we indeed see that GP-CCM statistic indicates that Rossler causes Lorenz dynamics over all states. CCM instead takes stronger coupling to indicate direction, and only in the case of X1 of the Rossler system does the coupling show towards all states of the Lorenz system.

However, results of increasing coupling for a Lorenz system driving a Rossler system are shown in Fig. 5. While there is a nonlinear relationship between the GP-CCM statistic $\kappa$ and $\epsilon$, the GP-CCM identifies all causal links for the states of Lorenz system driving the Rossler system; however, CCM performs poorly, unable to determine any directionality in any of the cases.

For both experiments performed, causal coupling from Lorenz system to Rossler system and vice versa, the GP-CCM statistic proved significant as outside the $95 \%$ confidence interval of the permutation analysis. An example of a statistical significance test can be seen in Fig. 13.

b. RL circuit with process noise. It can be seen in Fig. 6 that GP-CCM is far more robust to noise than CCM, as it is barely decreasing in strength of evidence of $V$ driving $I$ at increasing the noise contribution, whereas in CCM immediate presence of noise brings the values of the coupling statistic down to zero. This result illustrates the proposed Gaussian 


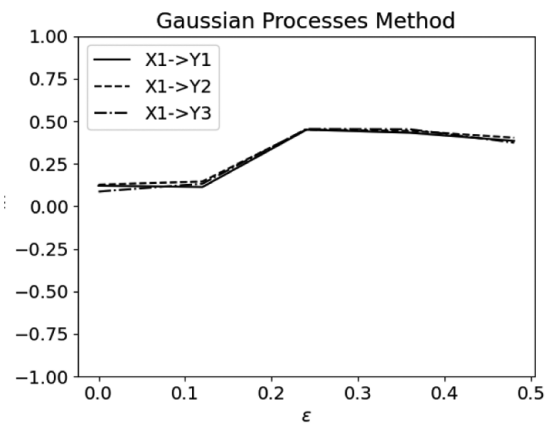

(a)

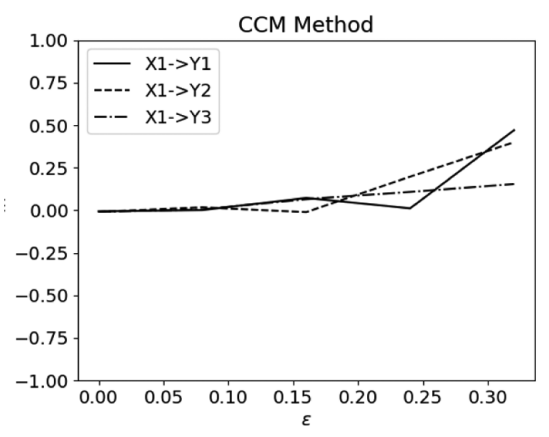

(d)

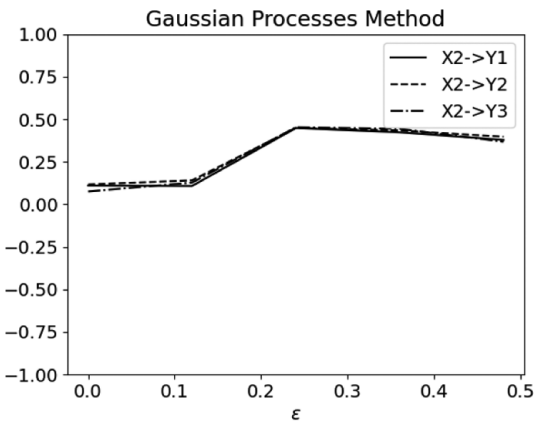

(b)

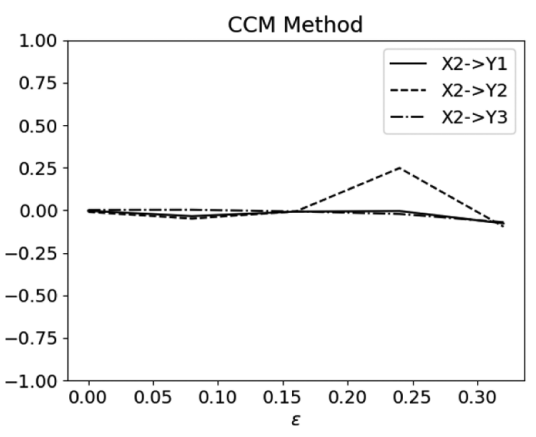

(e)

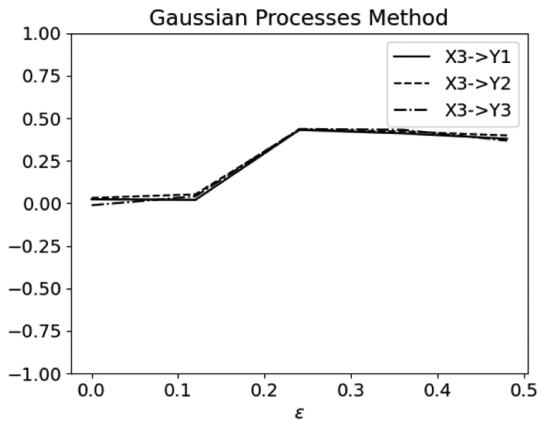

(c)

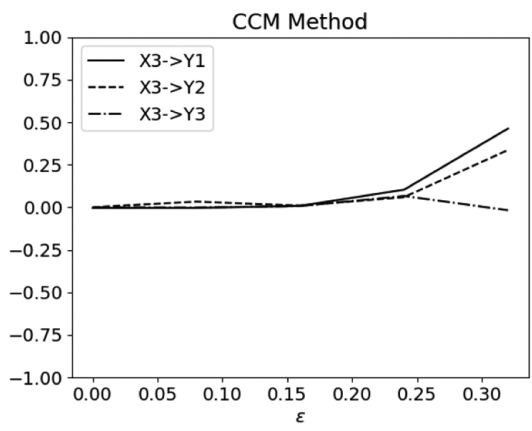

(f)

FIG. 4. Coupling statistics for CCM (d)-(f) and GP-CCM (a)-(c) plotted as a function of the coupling from the Rossler to the Lorenz state spaces modulated by the coupling parameter $\epsilon$ with a constant diffusion term.

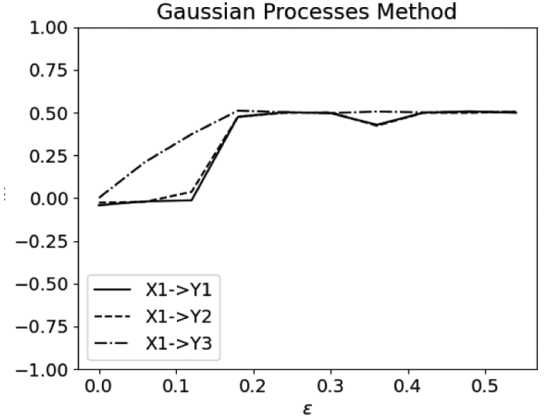

(a)

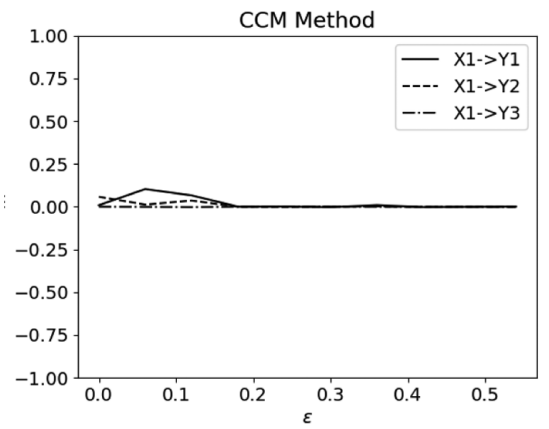

(d)

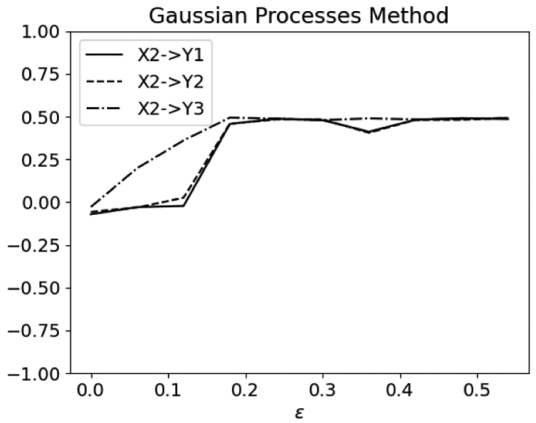

(b)

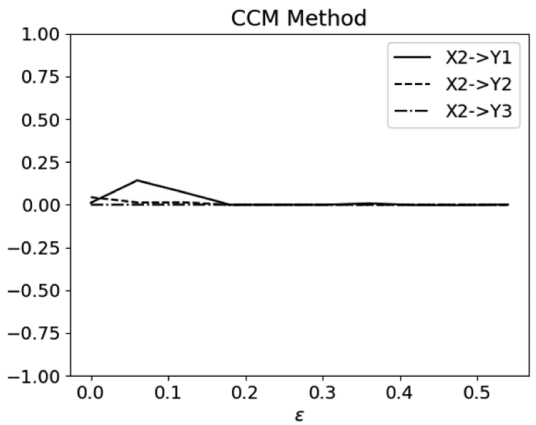

(e)

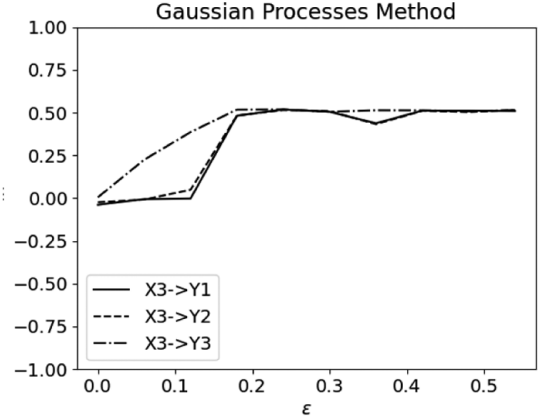

(c)

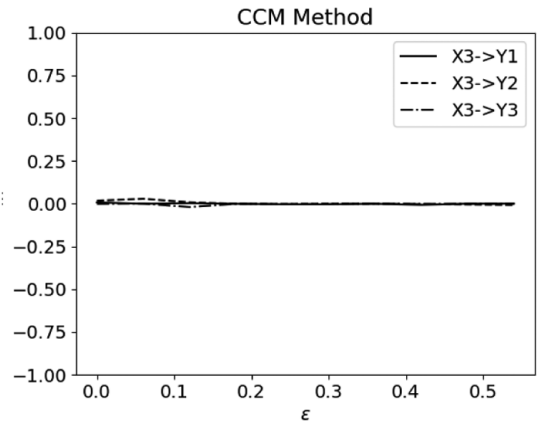

(f)

FIG. 5. Coupling statistics for CCM (d)-(f) and GP-CCM (a)-(c) plotted as a function of the coupling from the Lorenz to the the Rossler state spaces modulated by the parameter $\epsilon$ with a constant diffusion term. 


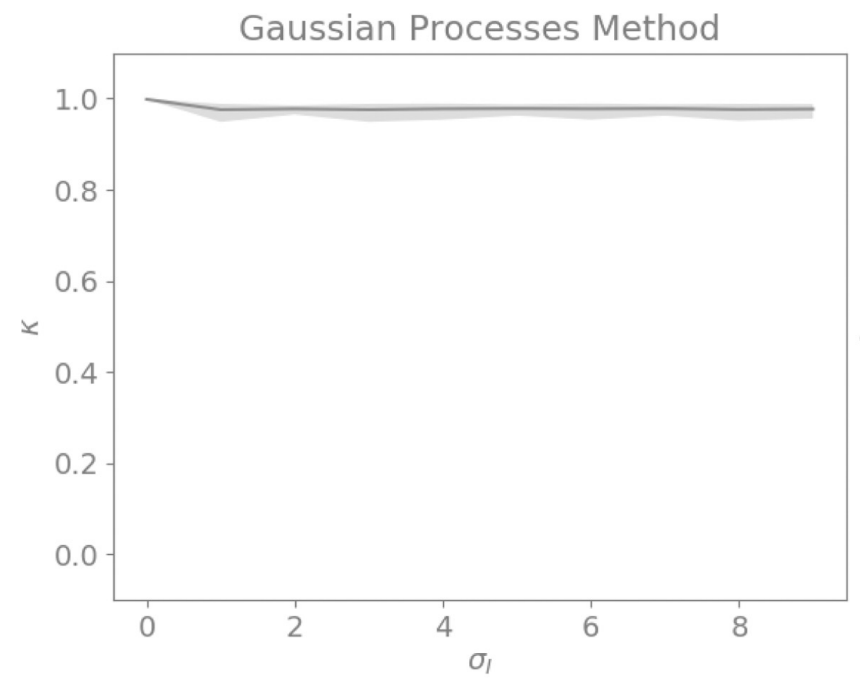

(a)

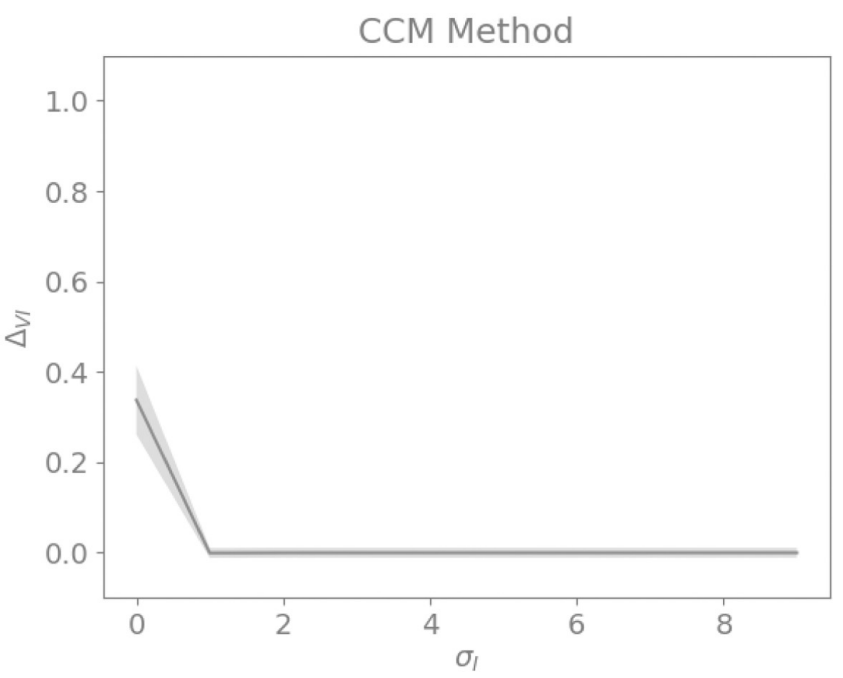

(b)

FIG. 6. Coupling statistics for GP-CCM (a) and CCM (b) computed between voltage and current in the simulated RL circuit as a function of the amount of noise on the current as illustrated in Eq. (23). The shaded area represents $95 \%$ confidence intervals over the realizations.

process method being robust to process noise, as well as the high sensitivity of CCM to stochastic disturbances on the deterministic dynamics.

\section{B. Physiological data}

a. Non-task-separated. Figure 7 shows that if we take the entire $10 \mathrm{~min}$ from each trial for causality analysis, GP-CCM consistently provides substantial evidence for the time series of each EEG band driving the fNIRS series. This result holds also when CCM is used, though the middle quartile presents some values that are negative, i.e., suggesting that fNIRS drives EEG. The GP-CCM, however, demonstrates that EEG is driving fNIRS in all bands, with stronger evidence in slower wave bands $\alpha, \theta$ and $\delta$. These results agree with the hypothesis as presented in Sec. 2.4.1 as well as the findings of Pfurtscheller et al., in which slow wave oscillations found in cardiac signals are strongly coupled with electrophysiology [56]. As it pertains to the comparison between the coupling statistic assessed for different EEG bandpowers, GP-CCM displays statistically significant differences between all pairs of distributions, while CCM detects differences only between the $\beta$ and $\gamma$ bands. This result suggests that the ability to identify EEG as the coupling direction with GP-CCM is bandspecific, with the dynamics of the EEG at lower frequencies $(\delta$ and $\theta$ ) providing a more clear indication of the EEG $\rightarrow$ fNIRS coupling as the prevalent one.

b. Task-separated. When the analysis is performed separating the baseline and task epochs in Fig. 8, we still see that EEG is the driver of the system for all bands in GPCCM, where all estimates display a median clearly above 0 . However, the strength of evidence is not as large as it was previously. This could suggest either a large uncertainty in state-space reconstruction due to the considerably shorter length of the analyzed time series (10 min down to $30 \mathrm{~s}$ ), or also the fact that NIRS and EEG may become slightly uncoupled at the moment of activation. The lower evidence of a coupling direction was observed also for CCM, where also we again see that it has higher variance than the GP-CCM further emphasizing the point made previously on its consistency. As discussed previously, the model of EEG with fNIRS in Ref. [57] suggests that the hemodynamic signal is directly caused by EEG. According to the model, the frequency profile shifts to higher frequencies upon activation and to lower frequencies upon deactivation; this, however, does not appear evident in either CCM or GPCCM, though summarily EEG appears to be the coupling direction.

\section{DISCUSSION AND CONCLUSIONS}

We have introduced a methodology for inferring causality in dynamical systems through Gaussian process theory. Leveraging both the concept of cross-mapping onto state spaces (see Fig. 9) and zero mean Gaussian process priors on functional spaces to create a Bayesian model evidence comparison between the causal strength computed separately along the directions $X \rightarrow Y$ and $Y \rightarrow X$, we have developed a robust metric for inferring coupling direction in noisy dynamical systems. We demonstrated the robustness of GP-CCM using synthetic data simulations first in bidirectional logistic maps without any process noise, to compare with a benchmark with known results; then in unidrectionally coupled chaotic systems with high order of complexity and indirect causal links, in order indirect causal links and show how increasing a forcing parameter induces stronger evidence of coupling; and finally an RL circuit to show that, even when the driving force presents simple dynamics, a clear driving direction can be determined even in the presence of process noise of high variation.

Having demonstrated the robustness of GP-CCM on the simulations performed, experiments were then performed on real physiological data. Interactions between complex systems in neuroscience provide an interesting setting for testing our method as it itself has high level of noise not only at the level of the dynamics, because uncertainty of metabolism dynamics 


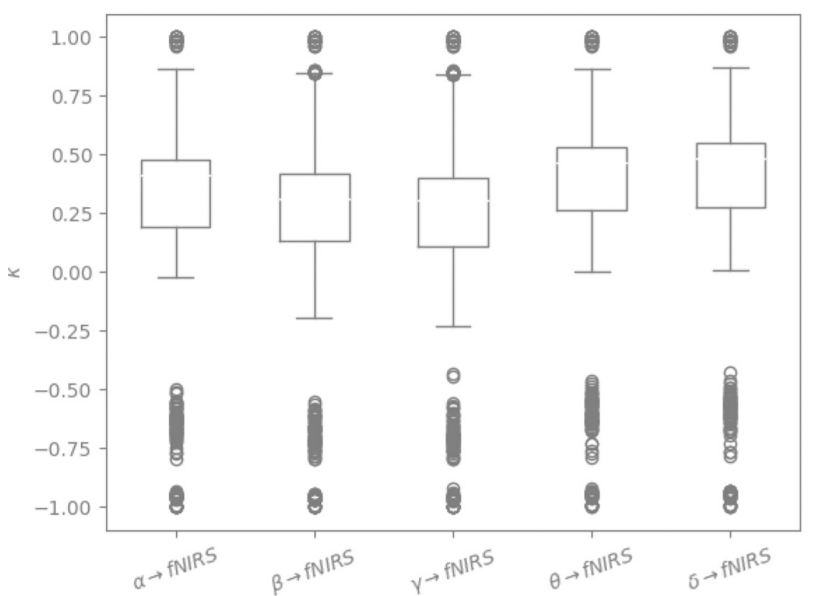

(a)

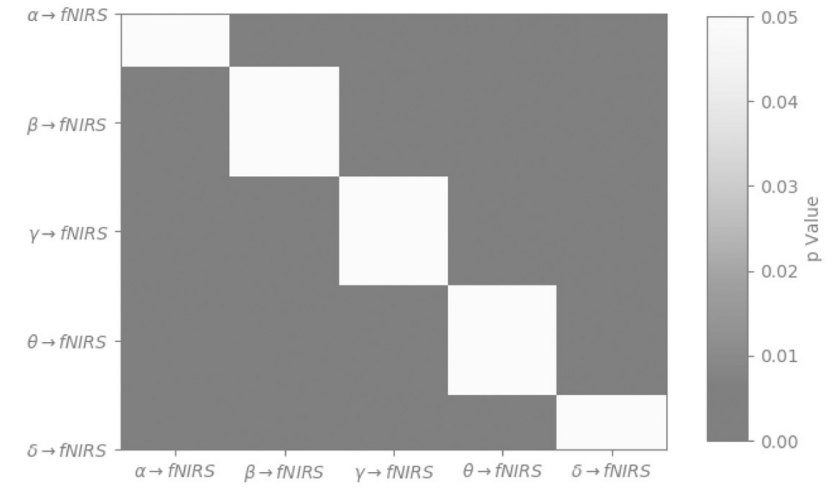

(c)

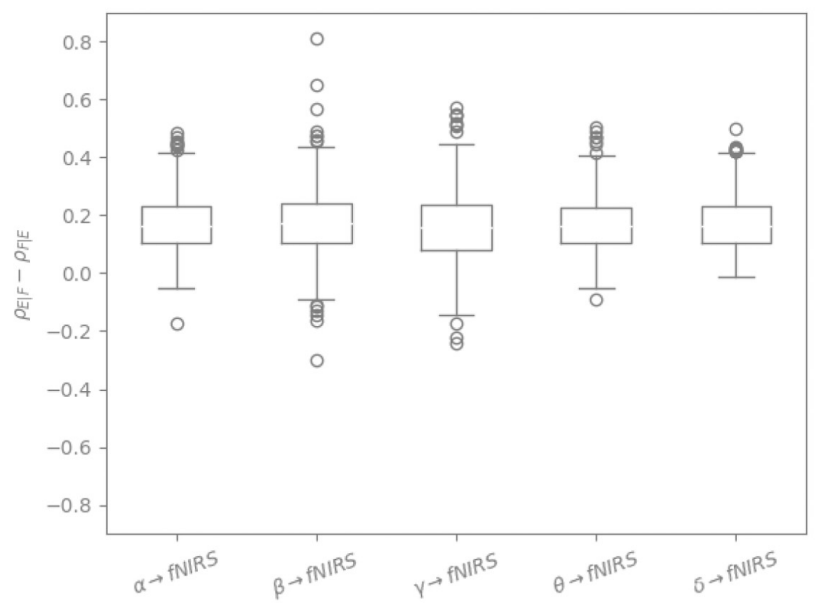

(b)

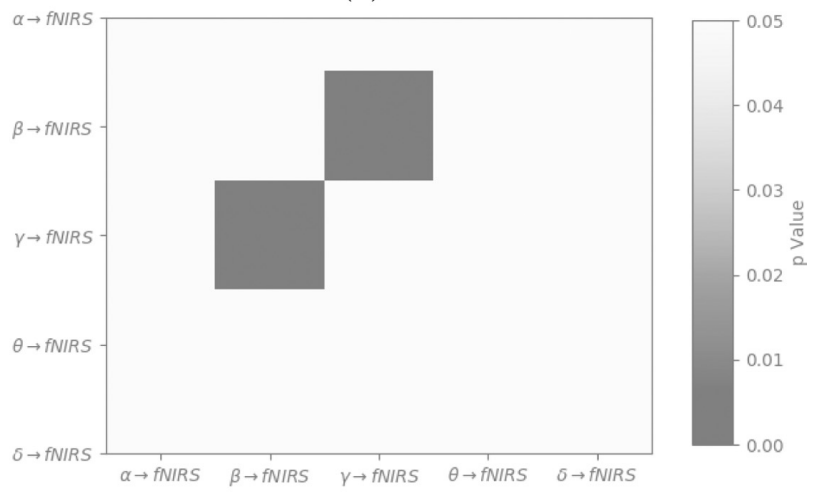

(d)

FIG. 7. Overall (non-task-separated) assessment of leading drivers in EEG-fNIRS coupling. The top panels report the distributions across subjects of the coupling statistic of GP-CCM and CCM computed at varying the EEG bandpower time series used for coupling analysis; positive values correspond to the case of EEG being the coupling direction. The bottom panels report the $p$ values of the Wilcoxon pairwise test, color-coded so that black and white represent statistically significant and nonsignificant comparisons.

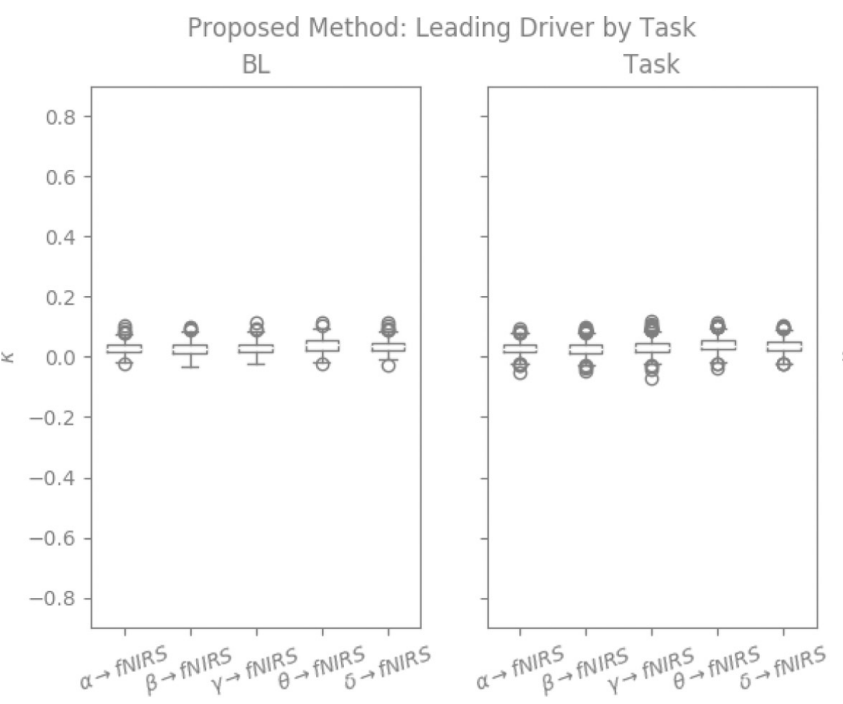

(a)

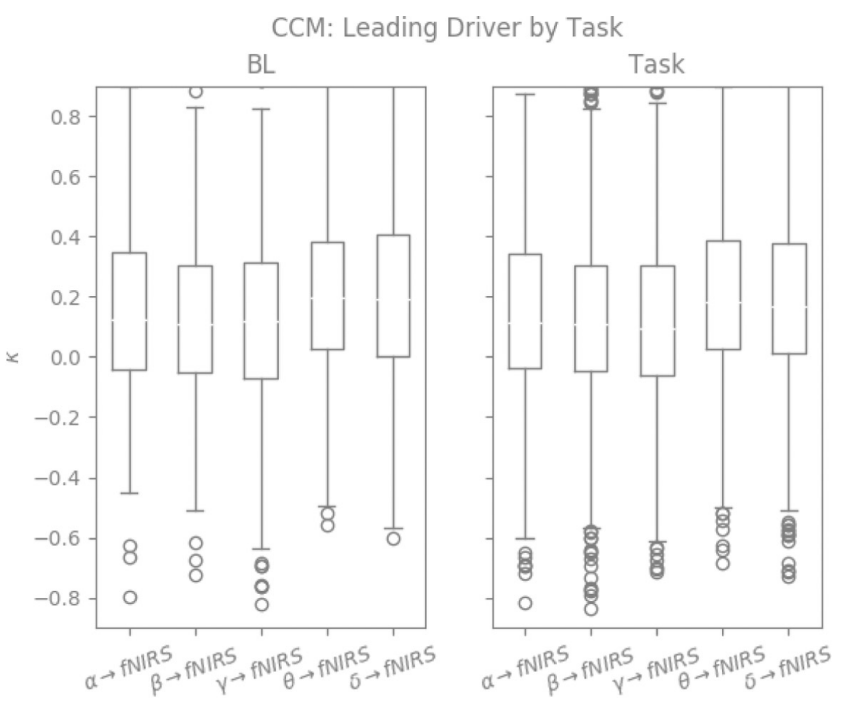

(b)

FIG. 8. Task-separated assessment of coupling direction in EEG-fNIRS coupling. Panels report the distributions across subjects of the coupling statistic of GP-CCM and CCM, computed separately for the baseline and task conditions at varying the EEG bandpower time series used for coupling analysis; positive values correspond to the case of EEG being the coupling direction. 
is being propagated by neural activity but also by extraneural activity. Also, on the topic of complex systems, neuroscience is a natural example of many body interactions such as the interactions seen in neurons. The acquisition of signals from these systems also have high degree of uncertainty with regards to the sensor systems themselves. We hypothesized that our GP-CCM not only provides more a consistent detection of causality compared to CCM, but also greater strength of evidence. Indeed we showed consistently prevailing causal strength in the direction of EEG $\rightarrow$ fNIRS, meaning that data shows more evidence that the electrophysiological activity is the leading causal driver of the metabolic, we also see that over realizations of a noise-driven dynamical system, when causality is strongly present, there is low variation in the statistic, giving confidence that our results are meaningful. On the topic of the decreasing causal strength observed when a subject performed a task, the additive noise term in the model as presented in Eq. (26) can possibly give explanatory power, demonstrating that predictability of fNIRS given the EEG decreases when there are extraneural activity present. Beyond that, a trove of literature has demonstrated how low frequency oscillations in hemodynamics demonstrate synchrony with EEG oscillations, reflecting potential forcing from hemodynamics on EEG oscillations [58,59]. Moreover, results of Ref. [58] demonstrated Granger causality for hemoglobin causing EEG to be higher than EEG to hemoglobin. Speculatively, this may be linked to the argument discussed by Ref. [12] that Granger causality suffers from poor separability in systems that are weak or moderately coupled systems as in the case of the bidirectional logistic maps (see Ref. [12] for further discussion). Importantly, the taskand time-dependence on the EEG-fNIRS causality may occur, especially in a closed loop fashion (i.e., electrophysiology continuously causes hemodynamics changes and vice versa). Nonetheless, further investigation needs to be performed to understand why this may be the case; in particular, a direct comparison between Granger causality implemented in the known form of predictability improvement from driver to target and cross prediction schemes implemented in the spirit of CCM from target to driver [60] is envisaged to investigate differences both in simulated and physiological data.

Furthermore, investigation needs to be done to truly parse why certain physiological oscillations are significantly different from others in determining the causal strength from activity to activity or region to region. A potential investigation in an extension of the mechanistic model presented in prior study that was used to create the hypotheses can be done to investigate such effects. For example, in the integrateand-fire function used to derive the first-order approximation, perhaps the relaxation time constant coefficient (which in the physical model is transmembrane conductance) can instead be looked into as a time varying function; presently, the firstorder approximation treats it as a constant, where the varying Jacobian is an approximation the nonlinear depolarization function $u$ seen in Eq. (25). The time-varying transmembrane conductance can reflect an extra variation of rate of change of electrical polarization.

In terms of extensions to the causal method proposed, it is possible that the signal may not be well characterized by Gaussian functions with a state space and covariance structure as we defined in this study. To that effect, future research should find an adequate map onto a zero-mean Gaussian process that preserves topological properties of the reconstructed phase space. This can be done by warping observation space via a basis expansion series as proposed in Ref. [61], where a neural networklike series of hyperbolic tangent functions in res-net fashion for invertibility was used. Another extension may comprise the use of invertible functions known as "normalizing flows" [62] to better approximate a true posterior from a base simple Gaussian density. Beyond that, one can investigate looking to deep Gaussian processes as another alternative to learn complex non-Gaussian spaces using compositions of Gaussian processes, where each layer has a zero-mean Gaussian prior placed on it [63].

From the simulations of a Lorenz system driving a Rossler system along with the inverse case, we have seen that the causal GP-CCM metric provides significant results at all coupling strength values. To that extent, future studies should focus on the development of a new statistical analysis devised ad hoc for uncoupled dynamical systems. For example, sensitivity analysis test on hyperparameters can be performed, or perhaps a latent model method could be interest to impose a distribution over the input parameters as in Ref. [64]. This may even further help substantiate results in Figs. 4 and 5 where we are on the fringe of the concept of "substantiality" of the metric proposed in Eq. (15), or for the physiological dataset where, even though we see a clear directionality, it is below the threshold for.

Though the results in Figs. 10 and 11 in the Appendix do show the ability to infer proper causal direction given nonstationary diffusion in the stochastic dynamics, the theory itself needs to have better founding for it. Indeed, the sparse kernel we derive does account for noise in variables by optimally finding points in state space that maximizes the marginal likelihood of our state space reconstruction, however no guarantees are particularly given with the theory. This, in turn, may improve the calculation in dynamical systems characterized by many timescales as physiological systems. For example, the method could be extended to a moving window method that considers short transient interactions alongside extended long-timescale analysis (see Fig. 12 for in the Appendix an example of a moving window analysis for a nonstationary diffusion signal). If not multiple timescales, then a covariance kernel composed by other nonstationary Gaussian processes such as the Wiener kernel could be utilized as well. This is also important to consider as pertaining to the task of deciding proper embedding dimension and delay times for mapping to time series to a reconstructed phase space; nonstationarity poses indeed inherent difficulties where the full system effects cannot be described in a single scale, requiring extended embedology analyses [65].

CCM and GP-CCM inherently focus on tackling the problem of synergistic nonlinear coupling in dynamical systems, which was cause of interest due to its prevelance in complex systems, e.g., such as neurophysical systems. As stated in Ref. [12], its predication is based on a system that explores its entire state space as sample size increases, creating a denser simplex for cross-mapping prediction, i.e., having a causal metric that converges. An inherent cause for concern, however, is the error in sampling times causing poor 
reconstructions of the state space with Takens' theorem. This may need to be further validated that it does not affect the proposed method. However, the proposed GP-CCM method appeared promisingly robust to observational and noise in dynamics, i.e., process noise as revealed in Figs. 4-6, which could be a sign of the robustness that the imposed prior imposes on inferring a posteriori causal direction.

It may be tempting from results such as Fig. 3 to make statements such as the predominant direction of coupling between bidirectional systems given our statistic. While this inference is not the main aim of the proposed methodology, we may elaborate on cyclic flow diagrams by determining which state space reconstruction has higher likelihood of cross mapping to another one. On that note, the statistical significance positive value of $\kappa_{Y X}$ can only make the claim of a single direction of information from $Y \rightarrow X$. To formally make wider predominant coupling direction statements for a bidirectional system, first we must develop an ad hoc statistic evaluating that there are indeed bidirectional interactions, i.e., that indeed there is also information flow from $X \rightarrow Y$; the additional mathematical formulation required for this inference can be a future case of study. Other tempting interpretations may be to look at, for example, $p\left(\phi(X) \mid \phi(Y), \theta_{y}^{*}\right)$ for a standardized metric of strength of information flow from $Y \rightarrow X$. We avoid such analysis as scale of the likelihood is impacted by the number of samples, e.g., for very large number of samples $\mathrm{N}$ and a diagonal posterior covariance the log likelihood is $\sum_{n=1}^{N} \log \left[p\left(\mu_{Y \mid X_{n}}, \Sigma_{Y \mid X_{n}}\right)\right]$, where if $p$ is strictly $<1$ the posterior likelihood would tend to $-\infty$, i.e., likelihood almost zero. Given that, we suggest the proposed method GP-CCM to be used for time series of equal sample size. However, we provide in the Appendix Fig. 14 to demonstrate the posterior likelihood and correlations maps used to construct Fig. 3.

Moreover, future works may also be directed toward the investigation of causal graphs via a set of conditional tests to see if information is gained from introducing another parameter into the reconstructed phase space, i.e., $M=\{X(t), X(t-$ $\tau), \ldots X(t-m \tau), Y(t), Y(t-\tau) \ldots Y(t-m \tau)\}$ to test causality on a third. Also, as mentioned previously, further a posterior analysis on the GP-CCM statistic with sensitivity analysis on hyperparameters, or probabilistic latent models, can be researched to address a rigorous power of statistic term. Beyond that, a thorough multiscale analysis using GP-CCM can be assessed to determine causality that addresses the full system effects.

\section{ACKNOWLEDGMENTS}

The research leading to these results has received partial funding from the European Commission Horizon 2020 Program under Grant Agreement No. 813234 of the project RHUMBO and by the Italian Ministry of Education and Research (MIUR) in the framework of the CrossLab project (Departments of Excellence).

\section{APPENDIX}

\section{Convergent cross mapping illustration}

Principles of convergent cross mapping leverage the diffeomorphic properties between state spaces reconstructed

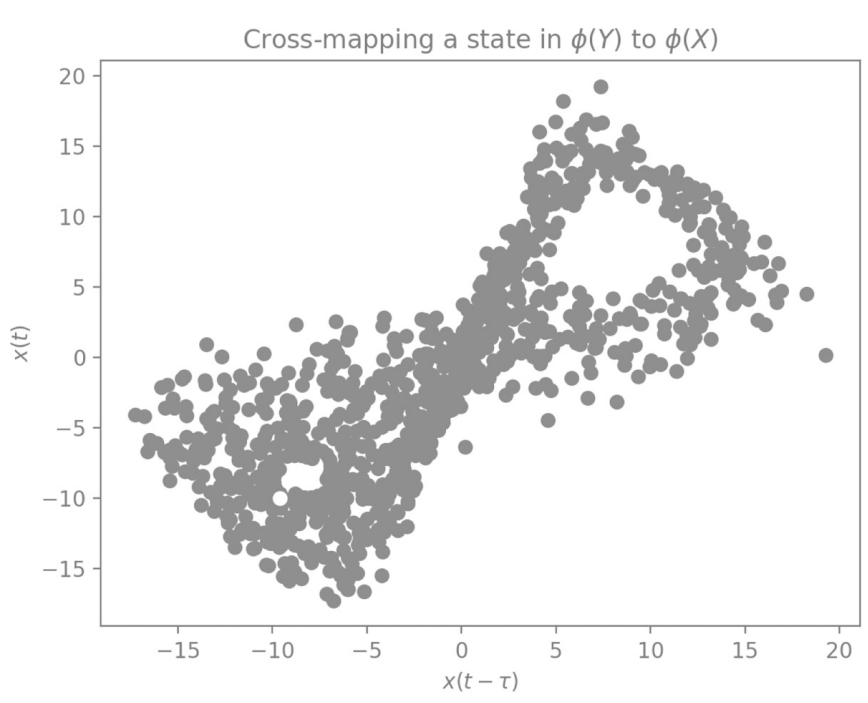

FIG. 9. Illustration of cross-mapping a single state from one time series onto a reconstructed attractor of a second time series. Taking a state as illustrated using a white dot (seen in the southwest corner of the plot) from a reconstructed phase space $X$ (full phase space in black) in a Lorenz system. $V$ is the three nearest neighbors to the white dot, as embedding dimension $m=2$; thus simplex $E=3 . W$ is then the distance to each of those three neighboring points $V$.

from single variable time series to the original state space of the dynamical system. If dynamical system $\mathcal{Y}$ reconstructed from observed time series $Y$ causes time series $X$, the delay-coordinate map of the driving time series $\phi(Y)$ should cross map well with high correlation onto the delay coordinate map $\phi(X)$ (see Fig. 9). The vice-versa, $\phi(X)$ cross-mapped onto the $\phi(Y)$ would not necessarily yield high correlation.

\section{Nonstationary noise effect on the statistics}

In order to assess the effect that nonstationary noise has on determining causality with either GP-CCM or CCM, we simulated the Rossler and Lorenz unidirectionally coupled systems in order to see whether the statistics are still able to uncover the proper directionality of coupling. Figs. 10 and 11 illustrate that indeed for GP-CCM we are still able to uncover the proper direction of causality for either variable of the system, though with a lower value than the stationary case seen in Figs. 4 or 5. CCM again seems to not be able to uncover any causal effect in the system coupling the Lorenz equations to the Rossler equations. For the Rossler equations driving Lorenz equations, CCM still provides inconsistent results.

\section{Sliding windows}

Sliding window techniques are popular for resolving potential transient and nonstationary phenomenon that occur in time series analysis that would not be able to be uncovered with only full window analysis of a time series. In Fig. 12 we 


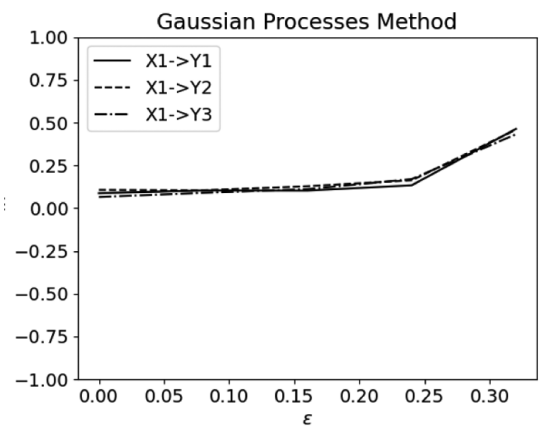

(a)

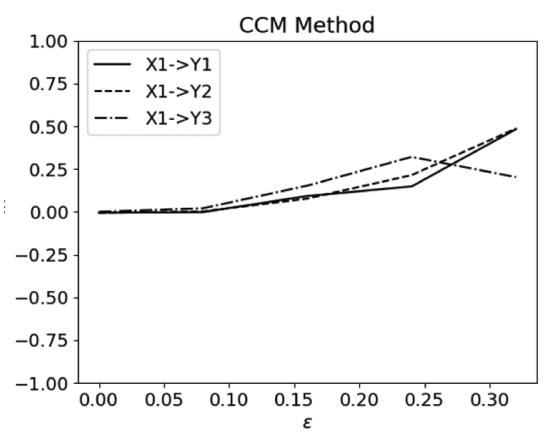

(d)

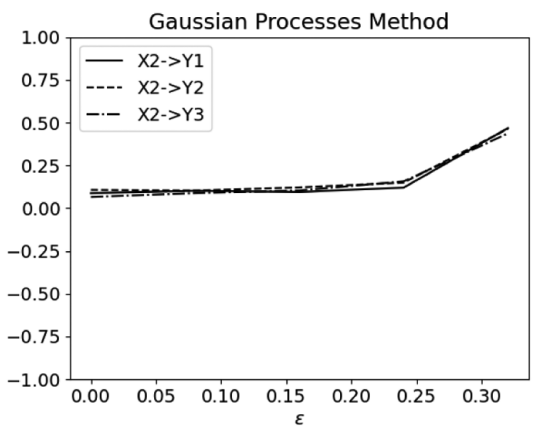

(b)

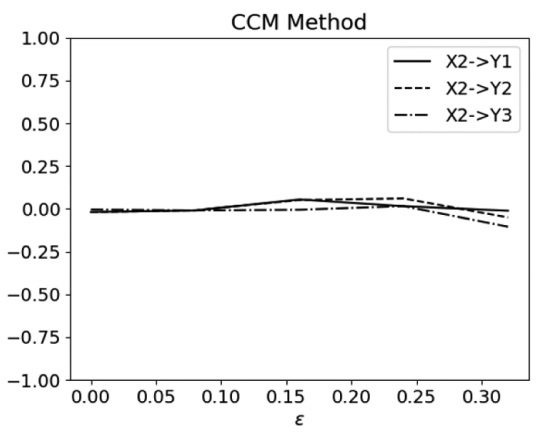

(e)

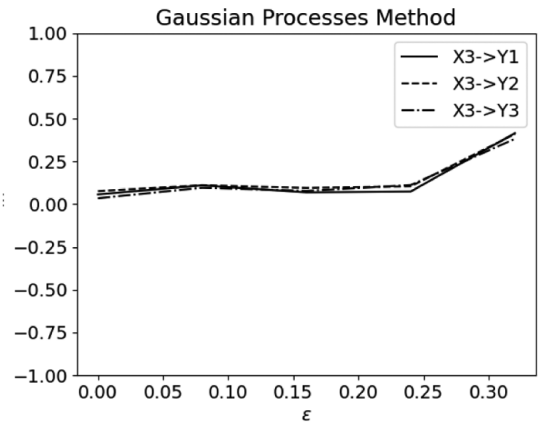

(c)

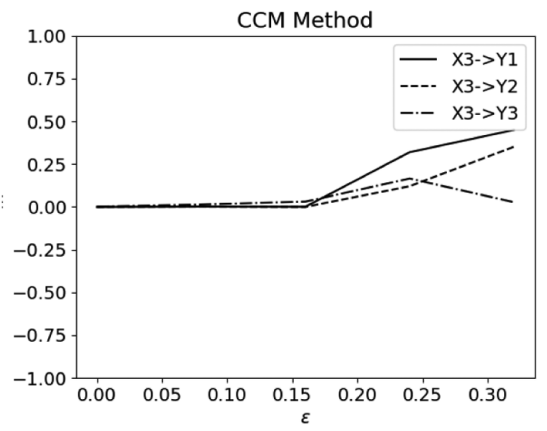

(f)

FIG. 10. Coupling statistics for CCM (d)-(f) and GP-CCM (a)-(c) plotted as a function of the coupling from the Rossler to the Lorenz system modulated by the coupling parameter $\epsilon$ with a noise diffusion term that linearly increases with time.

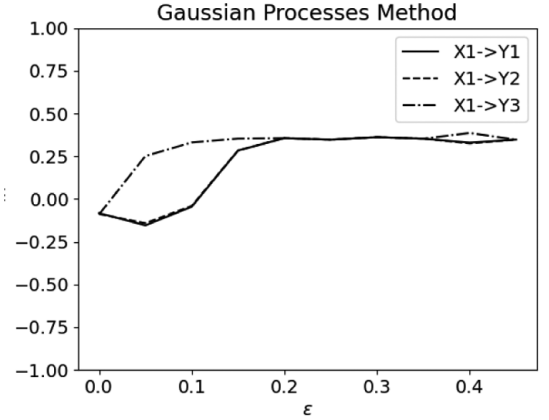

(a)

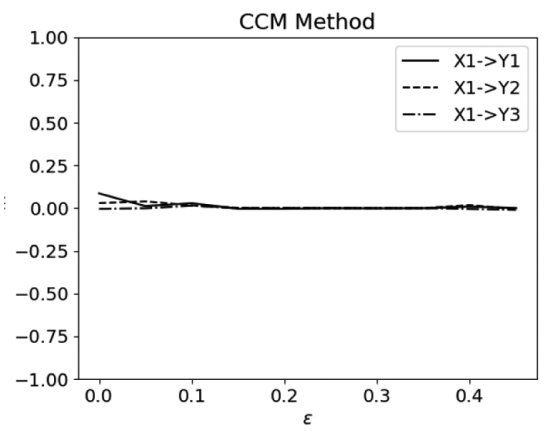

(d)

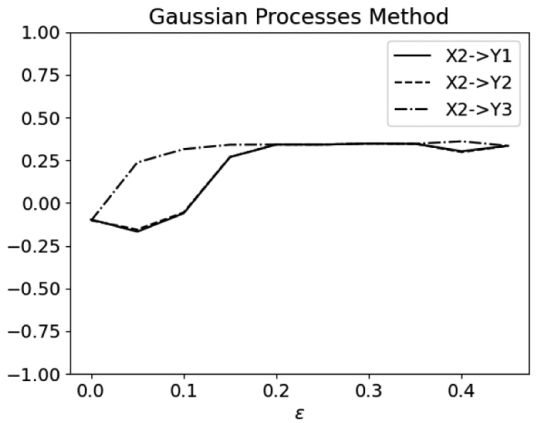

(b)

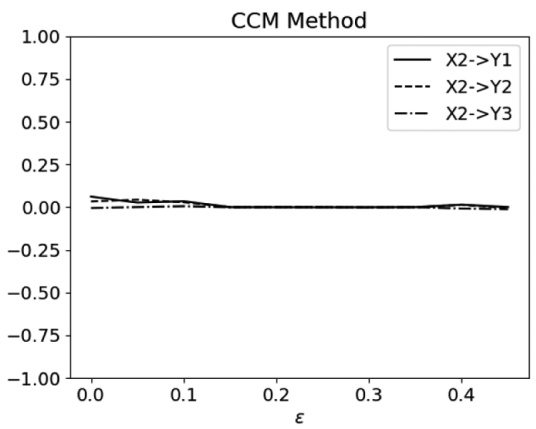

(e)

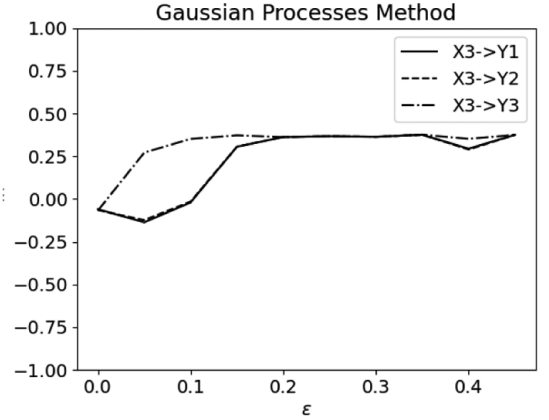

(c)

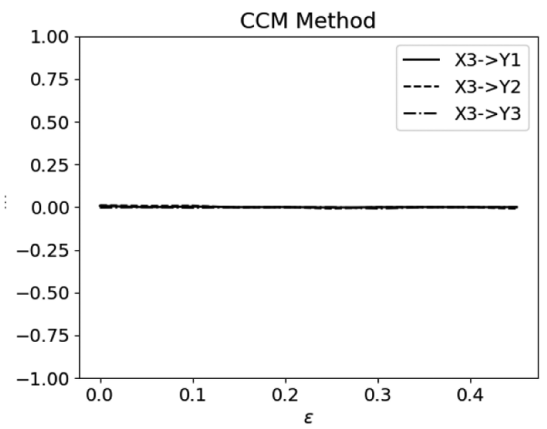

(f)

FIG. 11. Coupling statistics for CCM (d)-(f) and GP-CCM (a)-(c) plotted as a function of the coupling from the Lorenz to the the Rossler attractors modulated by the coupling parameter $\epsilon$ with a noise diffusion term that linearly increases with time. 


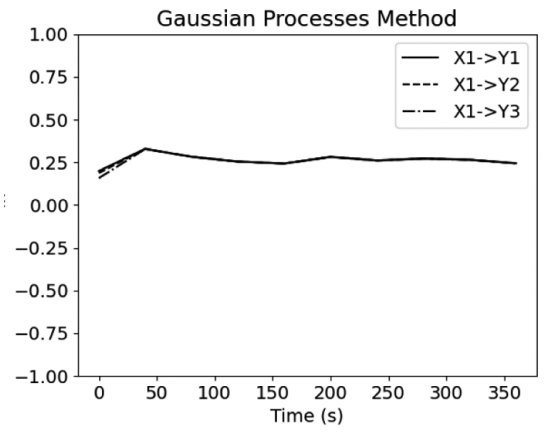

(a)

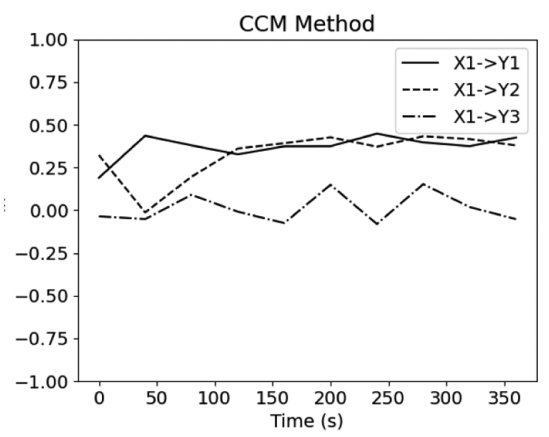

(d)

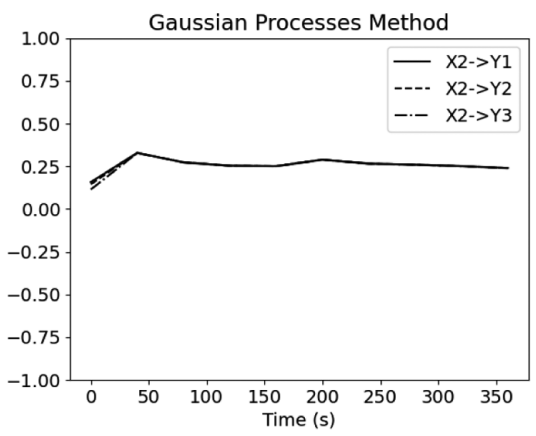

(b)

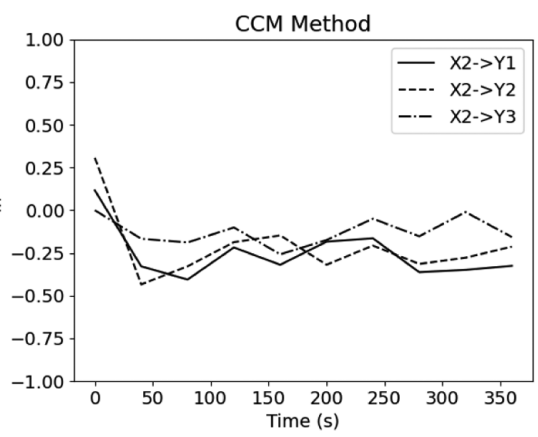

(e)

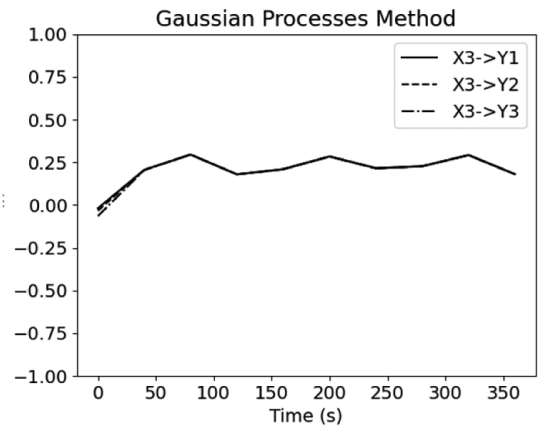

(c)

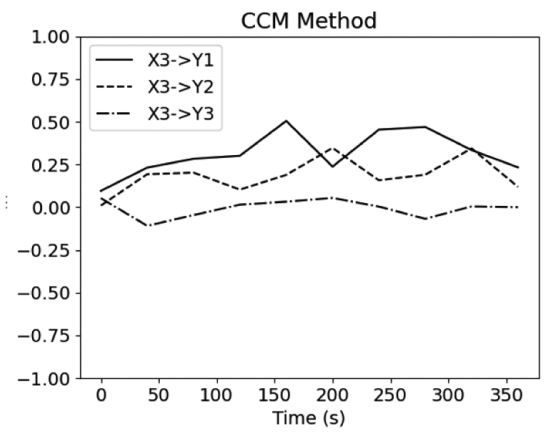

(f)

FIG. 12. Coupling statistics for CCM (d)-(f) and GP-CCM (a)-(c) plotted as a function of time from the Rossler to the Lorenz system modulated by the coupling parameter $\epsilon$ with a noise diffusion term that linearly increases with time. The physical coupling $\epsilon$ between Rossler to Lorenz systems was fixed to 0.3 as seen as the plateau of our causality graph in Fig. 10. Time series contain 4000 samples, thus the integration time is evenly spaced $0.1 \mathrm{~s}$. The window analysis for causality considers 400 samples with no window overlap. The function for diffusion is $g(t)=10^{-4} t$, so time zero has no diffusion, at end time the noise diffusion amplitude is 0.04 .

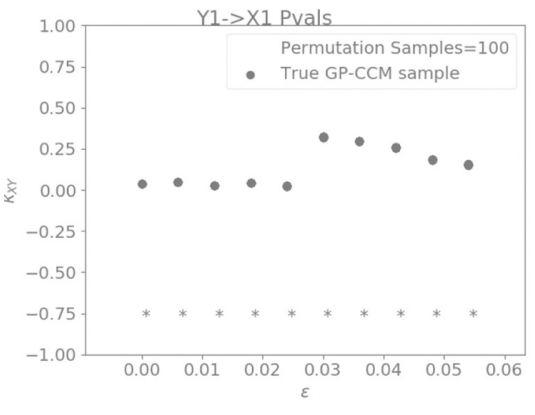

(a)

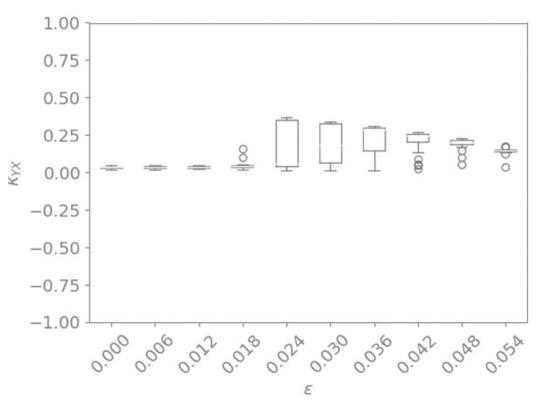

(d)

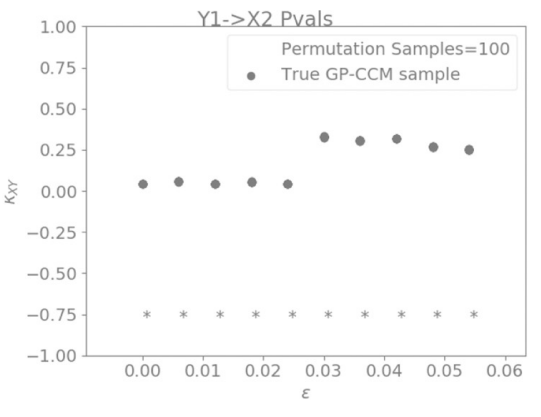

(b)

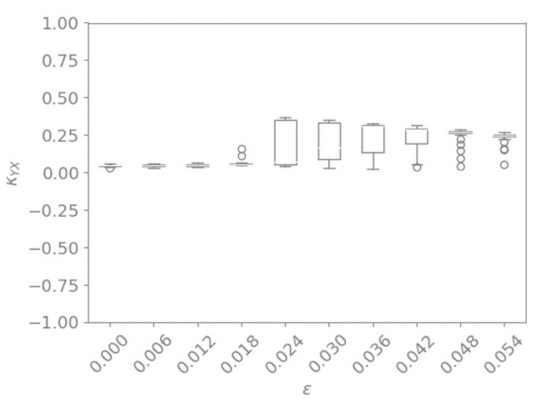

(e)

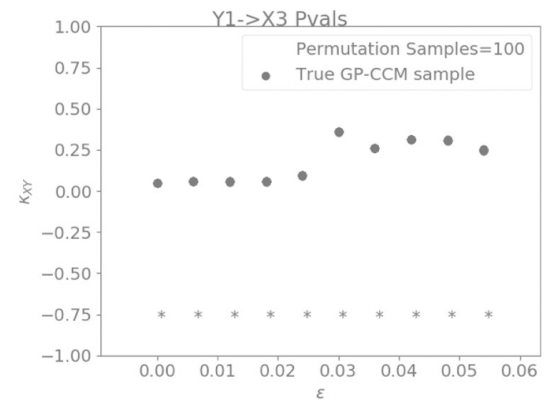

(c)

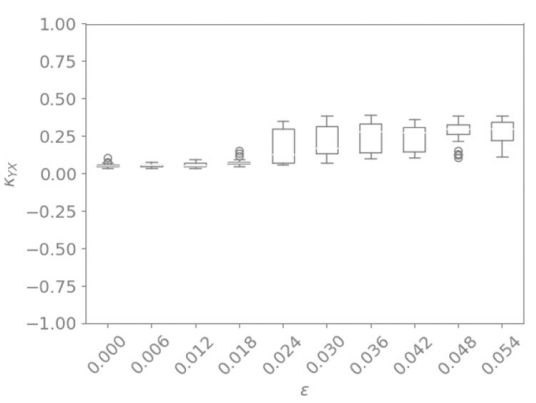

(f)

FIG. 13. Results of a surrogate data analysis testing whether the GP-CCM causal statistic did not arise from random chance, particularly for the Rossler state Y1 GP-CCM causing a Lorenz state $X$. In panels (a)-(c) the GP-CCM statistic is shown to be significant insofar as saying the statistic did not arise by random chance. Distance from the surrogate data increases as coupling parameter increases. In panels (d)-(f) boxplots of GP-CCM statistic over 30 realizations of coupled Rossler-Lorenz systems are shown, looking at the coupling direction between Rossler Y1 and each lorenz state $X$. 


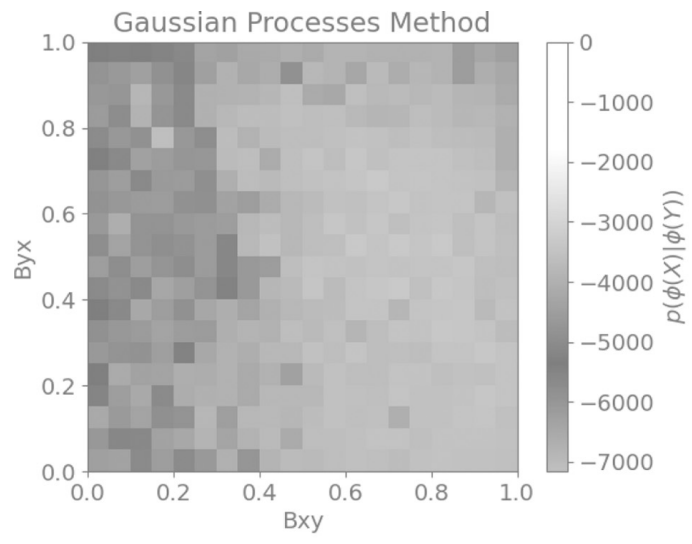

(a) $p(\phi(X) \mid \phi(Y))$

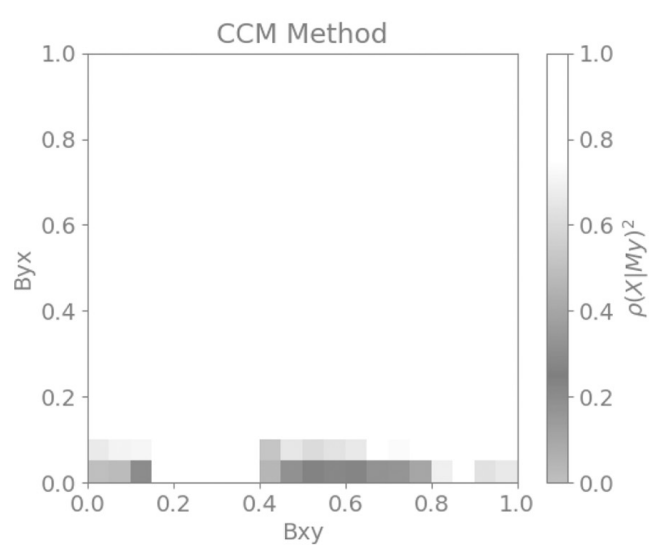

(c) $\rho_{X \mid \phi(Y)}$

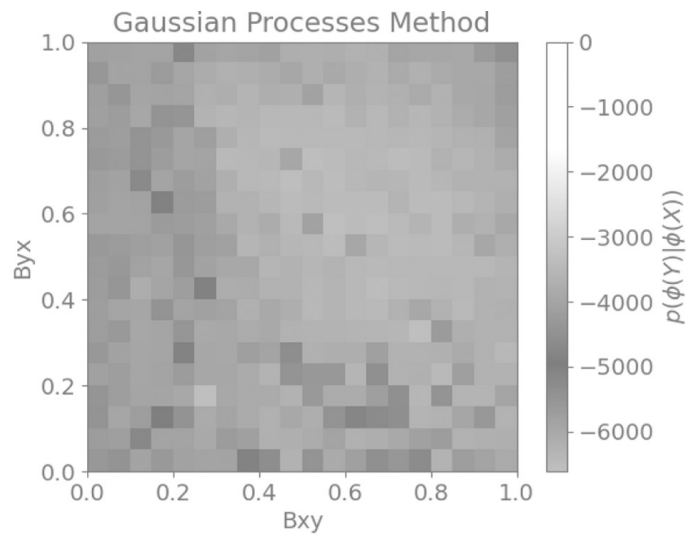

(b) $p(\phi(Y) \mid \phi(X))$

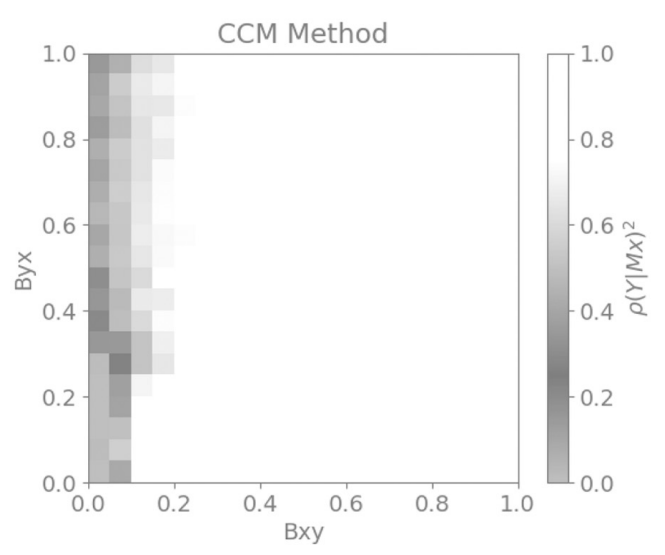

(d) $\rho_{Y \mid \phi(X)}$

FIG. 14. Heatmaps of posterior likelihoods in panels (a), (b) for the GP-CCM statistic and correlations in panels (c), (d) used to construct the $\kappa$ and $\Delta$ statistics in Fig. 3.

show results of GP-CCM and CCM on determining coupling direction using a sliding window technique.

\section{Surrogate data analysis}

In order to illustrate the surrogate data analysis for the GP-CCM method, we provide scatter-plots in Fig. 13 demonstrating for a single realization that the statistic will show significance, with distance from the surrogate data mean (i.e. 0 ) increasing as coupling strength increases. Furthermore, we illustrate the distribution of significant GP-CCM statistics over 30 realizations for each coupling strength value.

\section{Posterior likelihoods and correlations for Fig. 3}

The statistics in the heatmap Fig. 3, as illustrated in Eqs. (6) and (13) respectively for CCM and GP-CCM, are composed of intermediate statistics of correlations and posterior likelihoods. Fig. 14 illustrates the precise intermediate statistics used for constructing these heatmaps.
[1] T. Stankovski, T. Pereira, P. V. E. McClintock, and A. Stefanovska, Coupling functions: Universal insights into dynamical interaction mechanisms, Rev. Mod. Phys. 89, 045001 (2017).

[2] K. J. Friston, Functional and effective connectivity: A review, Brain Connect. 1, 13 (2011).

[3] H.-J. Park and K. Friston, Structural and functional brain networks: From connections to cognition, Science 342, 1238411 (2013).

[4] C. Granger, Investigating causal relations by econometric models and cross-spectral methods, Econometrica 37, 424 (1969).
[5] M. Kar, Şaban Nazlıoğlu, and H. Ağır, Financial development and economic growth nexus in the mena countries: Bootstrap panel granger causality analysis, Econ. Modell. 28, 685 (2011).

[6] S. Z. Chiou-Wei, C.-F. Chen, and Z. Zhu, Economic growth and energy consumption revisited - evidence from linear and nonlinear granger causality, Energy Econ. 30, 3063 (2008).

[7] T. Schreiber, Measuring Information Transfer, Phys. Rev. Lett. 85, 461 (2000).

[8] R. Vicente, M. Wibral, M. Lindner, and G. Pipa, Transfer entropy-A model-free measure of effective connectivity for the neurosciences, J. Comput. Neurosci. 30, 45 (2011). 
[9] A. Porta and L. Faes, Wiener-Granger causality in network physiology with applications to cardiovascular control and neuroscience, Proc. IEEE 104, 282 (2016).

[10] K. J. Friston, A. M. Bastos, A. Oswal, B. van Wijk, C. Richter, and V. Litvak, Granger causality revisited, NeuroImage 101, 796 (2014).

[11] M. Wibral, R. Vicente, and J. Lizier, Directed Information Measures in Neuroscience, Understanding Complex Systems (Springer, Berlin, Heidelberg, 2014).

[12] G. Sugihara, R. May, H. Ye, C.-h. Hsieh, E. Deyle, M. Fogarty, and S. Munch, Detecting causality in complex ecosystems, Science 338, 496 (2012).

[13] P. A. Corning, Synergy and self-organization in the evolution of complex systems, Syst. Res. 12, 89 (1995).

[14] K.J. Friston, A. Mechelli, R. Turner, and C.J. Price, Nonlinear responses in fmri: The balloon model, volterra kernels, and other hemodynamics, NeuroImage 12, 466 (2000).

[15] D. A. Smirnov and B. P. Bezruchko, Spurious causalities due to low temporal resolution: Towards detection of bidirectional coupling from time series, Europhys. Lett., 100, 10005 (2012).

[16] D. A. Smirnov, Spurious causalities with transfer entropy, Phys. Rev. E 87, 042917 (2013).

[17] P. A. Stokes and P. L. Purdon, A study of problems encountered in Granger causality analysis from a neuroscience perspective, Proc. Natl. Acad. Sci. USA 114, E7063 (2017).

[18] L. Barnett and A. K. Seth, Granger causality for state-space models, Phys. Rev. E 91, 040101(R) (2015).

[19] A. Wismüller, X. Wang, A. M. DSouza, and M. B. Nagarajan, A framework for exploring nonlinear functional connectivity and causality in the human brain: Mutual connectivity analysis (MCA) of resting-state functional MRI with convergent crossmapping and nonmetric clustering, arXiv:1407.3809.

[20] K. Schiecke, A. Schumann, F. Benninger, M. Feucht, K.-J. Baer, and P. Schlattmann, Brain-heart interactions considering complex physiological data: Processing schemes for time-variant, frequency-dependent, topographical and statistical examination of directed interactions by convergent cross mapping, Physiol. Meas. 40, 114001 (2019).

[21] A. K. Verma, A. Garg, A. Blaber, R. Fazel-Rezai, and K. Tavakolian, Analysis of causal cardio-postural interaction under orthostatic stress using convergent cross mapping, in 2016 38th Annual International Conference of the IEEE Engineering in Medicine and Biology Society (EMBC) (IEEE, 2016), pp. 23192322.

[22] L. Heskamp, A. Meel-van den Abeelen, J. Lagro, and J. Claassen, Convergent cross mapping: A promising technique for cerebral autoregulation estimation, Int. J. Clin. Neurosci. Ment. Health 1, S20 (2014).

[23] S. Parvaneh, S. Moharreri, S. Rezaei, and N. J. Dabanloo, Nonlinear causal cardiorespiratory interaction in response to color stimuli, Comput. Cardiol. 46, 1 (2019)..

[24] K. Schiecke, B. Pester, D. Piper, F. Benninger, M. Feucht, L. Leistritz, and H. Witte, Nonlinear directed interactions between HRV and EEG activity in children with TLE, IEEE Trans. Biomed. Eng. 63, 2497 (2016).

[25] A. T. Clark, H. Ye, F. Isbell, E. R. Deyle, J. Cowles, G. D. Tilman, and G. Sugihara, Spatial convergent cross mapping to detect causal relationships from short time series, Ecology 96, 1174 (2015).
[26] D. Mønster, R. Fusaroli, K. Tylén, A. Roepstorff, and J. F. Sherson, Causal inference from noisy time-series data - testing the convergent cross-mapping algorithm in the presence of noise and external influence, Future Gener. Comput. Syst. 73, 52 (2017).

[27] K. Friston, L. Harrison, and W. Penny, Dynamic causal modelling, NeuroImage 19, 1273 (2003).

[28] J. Runge, P. Nowack, M. Kretschmer, S. Flaxman, and D. Sejdinovic, Detecting and quantifying causal associations in large nonlinear time series datasets, Sci. Adv. 5, 4996 (2019).

[29] P. Spirtes, C. Glymour, and R. Scheines, Causation, Prediction, and Search, 2nd ed. (MIT Press, Cambridge, MA, 2000).

[30] J. Pearl, Causality: Models, Reasoning, and Inference (Cambridge University Press, Cambridge, UK, 2000).

[31] D. A. Smirnov, Quantification of causal couplings via dynamical effects: A unifying perspective, Phys. Rev. E 90, 062921 (2014).

[32] D. A. Smirnov, Transfer entropies within dynamical effects framework, Phys. Rev. E 102, 062139 (2020).

[33] G. Feng, J. G. Quirk, and P. M. Djurić, Detecting causality using deep Gaussian processes, in Proceedings of the 53rd Asilomar Conference on Signals, Systems, and Computers (2019), pp. 472-476.

[34] R. E. Kass and A. E. Raftery, Bayes factors, J. Am. Stat. Assoc. 90, 773 (1995).

[35] M. W. Barnett and P. M. Larkman, The action potential, Pract. Neurol. 7, 192 (2007).

[36] T. Kirschstein and R. Köhling, What is the source of the EEG? Clin. EEG Neurosci. 40, 146 (2009).

[37] R. Plonsey and D. B. Heppner, Considerations of quasistationarity in electrophysiological systems, Bull. Math. Biophys. 29, 657 (1967).

[38] P. Magistretti and I. Allaman, A cellular perspective on brain energy metabolism and functional imaging, Neuron 86, 883 (2015).

[39] S. Ogawa, T. M. Lee, A. R. Kay, and D. W. Tank, Brain magnetic resonance imaging with contrast dependent on blood oxygenation, Proc. Nat. Acad. Sci. USA 87, 9868 (1990).

[40] T. Durduran, R. Choe, W. B. Baker, and A. G. Yodh, Diffuse optics for tissue monitoring and tomography, Rep. Prog. Phys. Phys. Soc. 73, 076701 (2010).

[41] F. Takens, Detecting strange attractors in turbulence, in Dynamical Systems and Turbulence, Warwick 1980, edited by D. Rand and L.-S. Young (Springer, Berlin, 1981), pp. 366-381.

[42] T. Sauer, J. A. Yorke, and M. Casdagli, Embedology, J. Stat. Phys. 65, 579 (1991).

[43] C. E. Rasmussen and C. K. I. Williams, Gaussian Processes for Machine Learning (Adaptive Computation and Machine Learning) (MIT Press, Cambridge, MA, 2005).

[44] D. J. C. MacKay, Bayesian methods for backpropagation networks, in Models of Neural Networks III: Association, Generalization, and Representation, edited by E. Domany, J. L. van Hemmen, and K. Schulten (Springer, New York, NY, 1996), pp. 211-254.

[45] E. Snelson and Z. Ghahramani, Sparse Gaussian processes using pseudo-inputs, in Proceedings of the 18th International Conference on Neural Information Processing Systems, NIPS'05 (MIT Press, Cambridge, MA, 2005), pp. 1257-1264. 
[46] A. C. Damianou, M. K. Titsias, and N. D. Lawrence, Variational inference for latent variables and uncertain inputs in Gaussian processes, J. Mach. Learn. Res. 17, 1 (2016).

[47] Y. Gal, M. v. d. Wilk, and C. E. Rasmussen, Distributed variational inference in sparse Gaussian process regression and latent variable models, in Proceedings of the 27th International Conference on Neural Information Processing Systems, NIPS'14 (MIT Press, Cambridge, MA, 2014), pp. 3257-3265.

[48] E. Snelson and Z. Ghahramani, Variable noise and dimensionality reduction for sparse Gaussian processes, in Proceedings of the 22nd Conference on Uncertainty in Artificial Intelligence, UAI'06 (AUAI Press, Arlington, VA, 2006), pp. 461-468.

[49] H. D. I. Abarbanel and M. B. Kennel, Local false nearest neighbors and dynamical dimensions from observed chaotic data, Phys. Rev. E 47, 3057 (1993).

[50] J. Shin, A. von Lühmann, B. Blankertz, D. W. Kim, J. Jeong, H. J. Hwang, and K. R. Müller, Open access dataset for eeg+nirs single-trial classification, IEEE Trans. Neural Syst. Rehabil. Eng. 25, 1735 (2017).

[51] P. Pinti, F. Scholkmann, A. Hamilton, P. Burgess, and I. Tachtsidis, Current status and issues regarding pre-processing of FNIRs neuroimaging data: An investigation of diverse signal filtering methods within a general linear model framework, Front. Human Neurosci. 12, 505 (2019).

[52] G. Strangman, D. A. Boas, and J. P. Sutton, Non-invasive neuroimaging using near-infrared light., Biol. Psychiatry 52, 679 (2002).

[53] M. Junghöfer, T. Elbert, D. Tucker, and B. Rockstroh, Statistical control of artifacts in dense array eeg/meg studies, Psychophysiology 37, 523 (2000).

[54] L. J. Gabard-Durnam, A. S. Mendez Leal, C. L. Wilkinson, and A. R. Levin, The Harvard automated processing pipeline for electroencephalography (Happe): Standardized processing software for developmental and high-artifact data, Front. Neurosci. 12, 97 (2018).

[55] E. Başar and B. Güntekin, Chapter 19-Review of delta, theta, alpha, beta, and gamma response oscillations in neuropsychiatric disorders, in Application of Brain Oscillations in Neuropsychiatric Diseases, Supplements to Clinical Neurophysiology, edited by E. Başar, C. Başar-Eroĝlu, A. Özerdem, P. Rossini, and G. Yener (Elsevier, Amsterdam, 2013), Vol. 62, pp. 303-341.
[56] G. Pfurtscheller, A. R. Schwerdtfeger, A. Seither-Preisler, C. Brunner, C. Stefan Aigner, J. Brito, M. P. Carmo, and A. Andrade, Brain-heart communication: Evidence for "central pacemaker" oscillations with a dominant frequency at $0.1 \mathrm{~Hz}$ in the cingulum, Clin. Neurophysiol. 128, 183 (2017).

[57] J. M. Kilner, J. Mattout, R. Henson, and K. J. Friston, Hemodynamic correlates of eeg: A heuristic, NeuroImage 28, 280 (2005).

[58] P. Lachert, J. Zygierewicz, D. Janusek, P. Pulawski, P. Sawosz, M. Kacprzak, A. Liebert, and K. J. Blinowska, Causal coupling between electrophysiological signals, cerebral hemodynamics and systemic blood supply oscillations in Mayer wave frequency range, Int. J. Neural Syst. 29, 1850033 (2019).

[59] G. Pfurtscheller, I. Daly, G. Bauernfeind, and G. R. MüllerPutz, Coupling between intrinsic prefrontal $\mathrm{HBO} 2$ and central eeg beta power oscillations in the resting brain, PLoS One 7, e43640 (2012).

[60] L. Faes, A. Porta, and G. Nollo, Mutual nonlinear prediction as a tool to evaluate coupling strength and directionality in bivariate time series: Comparison among different strategies based on $k$ nearest neighbors, Phys. Rev. E 78, 026201 (2008).

[61] E. Snelson, C. Rasmussen, and Z. Ghahramani, Warped Gaussian processes, in Advances in Neural Information Processing Systems 16, Max-Planck-Gesellschaft (MIT Press, Cambridge, MA, 2004), pp. 337-344.

[62] D. Rezende and S. Mohamed, Variational inference with normalizing flows, in Proceedings of the 32nd International Conference on Machine Learning, edited by F. Bach and D. Blei (PMLR, Lille, France, 2015), Vol. 37, pp. 1530-1538.

[63] A. Damianou and N. Lawrence, Deep Gaussian processes, in Proceedings of the 16th International Conference on Artificial Intelligence and Statistics, edited by C. M. Carvalho and P. Ravikumar (PMLR, Scottsdale, AZ, 2013), Vol. 31, pp. 207215.

[64] M. Titsias and N. D. Lawrence, Bayesian Gaussian process latent variable model, in Proceedings of the 13th International Conference on Artificial Intelligence and Statistics, edited by Y. W. Teh and M. Titterington (PMLR, Chia Laguna Resort, Sardinia, Italy, 2010), Vol. 9, pp. 844-851.

[65] R. Manuca and R. Savit, Stationarity and nonstationarity in time series analysis, Physica D 99, 134 (1996). 